\title{
Aerostructural design optimization of a 100-passenger regional jet with surrogate-based mission analysis
}

\author{
Rhea P. Liem*, Charles A. Mader ${ }^{\dagger}$ Edmund Lee ${ }^{\dagger}$ \\ University of Toronto Institute for Aerospace Studies, Toronto, ON, Canada \\ Joaquim R. R. A. Martins $\ddagger$ \\ University of Michigan, Department of Aerospace Engineering, Ann Arbor, MI, USA
}

\begin{abstract}
In this paper we present a coupled aerostructural optimization procedure for the design of a fuelefficient regional aircraft configuration. A detailed mission analysis is performed on an optimized flight mission profile to accurately compute the mission range, fuel burn, and flight time. The mission analysis procedure is designed to allow flexible mission profiles including those with a variety of cruise, climb and descent segments in the profile. The direct operating cost (DOC) is computed based on the mission characteristics (fuel weight, range, and time), and is then used as the objective function in the optimization problem. We use a coupled aerostructural solver comprised of a high-fidelity structural solver and medium-fidelity aerodynamic solver to solve for the static aeroelastic shape of the lifting surfaces. Due to the large computational cost associated with these solvers, "kriging with a trend" surrogate models are employed to approximate the aerodynamic force and moment coefficients required in the mission analysis. This approach is demonstrated in two DOC minimization cases: a mission profile optimization with a fixed geometry, and an aerostructural optimization with fixed, previously optimized mission profiles for a 100-passenger regional jet aircraft.
\end{abstract}

\section{Introduction}

Aircraft fuel consumption is expected to keep increasing in the next few decades as air traffic continues to grow [1]. With fuel prices consistently increasing over the past few years [2], total aviation fuel use now contributes significantly to the total aircraft operating costs and prices. In addition, fuel consumption is a direct surrogate for $\mathrm{CO}_{2}$ emissions, which is an important greenhouse gas, and also an indirect indicator for other aircraft emissions, such as $\mathrm{NO}_{\mathrm{x}}$ and $\mathrm{H}_{2} \mathrm{O}$ [3]. Due to these growing environmental concerns and the challenging fuel economics of aircraft, the appropriate fuel economy improvement goals for future aircraft need to be prudently selected from the available technological improvement options and policy scenarios. To assist such decision making and policy analysis processes, optimization techniques are often employed. However, to perform the optimization properly, first and foremost the right objective function needs to be carefully selected. Several objective functions have been proposed in previous work, such as maximum takeoff weight (MTOW), direct operating cost (DOC) [4], and block fuel (the minimum fuel mass for the fixed range) [5]. Minimizing MTOW pushes both the acquisition costs and the operational costs lower, as fuel weight is incorporated in the objective [6]. Wakayama [7] and Liebeck [8] successfully demonstrate this optimization strategy to design the BWB concept on a coupled system. Kenway et al. [9] demonstrate and compare high-fidelity aerostructural optimization problems with fuel burn and MTOW as the objective functions. Kennedy and Martins [10] use as a design objective a weighted combination of the takeoff gross-weight (TOGW), as a rough surrogate for the overall aircraft acquisition cost, and fuel burn, which contributes significantly to the aircraft's DOC. By assigning more weight on the fuel burn objective, they obtain optimum designs with increasing importance on the aerodynamic performance of the aircraft.

In this work, DOC is used as the objective function since it captures many aspects of aircraft operation, such as fuel burn, fuel price, flight range, block time, and MTOW. The effect of those factors on the net change in DOC is not straightforward, due to their interaction with each other. For example, increasing the cruise speed consequently reduces block time, which reduces DOC, but increases fuel burn, which causes DOC to go up. The right tradeoff is thus required for a net decrease in DOC. Fuel price determines how the fuel cost component weighs in the DOC computation. Higher fuel price calls for a configuration with reduced fuel burn, producing a more aerodynamically efficient aircraft with lower drag.

To compute DOC, it is critical to accurately and efficiently estimate the total fuel burn, mission range, and time of aircraft missions. An accurate calculation of the mission performance requires modeling the aircraft operation as

\footnotetext{
*Ph.D. Candidate, AIAA Student Member

${ }^{\dagger}$ Research associate, AIAA Member

${ }^{\ddagger}$ Associate Professor, AIAA Associate Fellow
} 
realistic as possible, including the mission profile, aircraft weights and balance, and aircraft engine. To analyze the aircraft performance, it is important to consider both aerodynamic and structural disciplines simultaneously, to account for the interaction and tradeoffs between the two disciplines, for which we use a multidisciplinary design optimization (MDO) framework. An MDO framework is an efficient technique to assist the design of engineering systems, as it takes into account the coupling in the system and automatically performs the optimal interdisciplinary trade-offs [11].

The classical Breguet range equation is commonly used to compute the amount of fuel burn during flight $[3,12,13]$. This widely used range equation was derived and published independently in 1920 by Coffin [14] and later in 1923 by Breguet [15]. This equation has since become a basic model describing the physics of aircraft, encompassing the three dominant disciplines within an aircraft system: engine (by the thrust specific fuel consumption or TSFC), aerodynamics (by the lift to drag ratio, $L / D$ ), and structural technologies (by the structural weight). This equation, however, is only applicable under the assumption that TSFC, $L / D$, and flight speed are constant. One important implication is that the takeoff, climb, and descent segments are not properly modeled by this equation [16]. Simple fuel fractions are typically used to compute the amount of fuel burned in flight segments other than cruise. A fuel fraction is defined as the ratio of the aircraft total weight at the end of a flight segment to the weight at the start of the same segment. See, for example, Roskam [13] for values of suggested fuel-fractions corresponding to several mission phases for various aircraft types. A combined factor is also sometimes used. For example, the fuel burn for taxi, takeoff, approach and landing can be collectively approximated by taking a fraction of takeoff weight; typically a factor of 0.007 is used for the total of those phases [17].

Lee and Chatterji [18] present the approximation functions for total fuel burn in climb, cruise, and descent phases. To compute fuel burn during climb, they apply a climb fuel increment factor, which is defined as the additional fuel required to climb the same distance as it is for cruise, normalized with respect to the takeoff weight [17]. This climb fuel increment factor is expressed as a quadratic polynomial function of cruise altitude and cruise speed with different sets of coefficients, depending on the aircraft type. Henderson et al. [19] present an object-oriented aircraft conceptual design toolbox, PYACDT, which analyzes a given mission profile to estimate the mission fuel burn and point performance parameters. The Breguet range equation is used to calculate the cruise range. This toolbox uses a potential flow panel method for its aerodynamic module. The Program for Aircraft Synthesis Studies (PASS), created by Desktop Aeronautics, Inc., is a conceptual design tool which evaluates all aspects of mission performance [20]. This software package can incorporate several analyses, including linear aerodynamic models for lift and inviscid drag, sonic boom prediction for supersonic cases, weight and CG estimation, and full mission analysis. These rapid analyses are coupled with optimization tools (gradient or non-gradient based) to perform aircraft design optimizations.

The fuel burn computations mentioned above are done with simplificatons of the aircraft performance and mission profile. This simplification might lead to inaccurate prediction of the total aircraft fuel consumption. For example, the constant $L / D$, TSFC, and flight speed assumed in the Breguet range equation do not reflect the actual aircraft operation, as their values vary across the flight operating points in the mission profile. This assumption in turn leads to inaccurate calculation of fuel burn, mission range and time, and thus an inaccurate DOC. Moreover, most fuel burn computation focuses more on the cruise portion, which is critical for long range missions, but not necessarily so for shorter range missions. For shorter range missions, the climb segments will contribute significantly to the total fuel consumption as well. Though desirable, performing a detailed mission analysis could be computationally expensive due to the thousands of performance evaluation needed to model all flight operating points in a mission. Solving an optimization problem with such expensive analyses can quickly become computationally prohibitive. Analytical and empirical models are sometimes used to reduce the computational time [21], in the expense of accuracy. Liem et al. [22] develop a mission analysis procedure to compute aircraft fuel burn using kriging surrogate models to approximate the aerodynamic performance. The use of surrogate models help reducing the computational cost significantly. However, the detailed analysis is still only limited to the cruise segment.

The main objective of this work is to develop an integrated toolbox to perform a detailed mission analysis that models all the climb, cruise, and descent segments. An engine model is employed to compute the TSFC value at each flight operating point, and a weight and balance model is used to estimate the weight and centre of gravity (CG) location of each component. Given a mission profile, aircraft geometry and engine specifications, this toolbox will compute the total fuel burn and DOC of the mission. To make this procedure more computationally tractable, "kriging with a trend" surrogate models are used to approximate the aerodynamic force and moment coefficients required in the mission computation.

To demonstrate the capability of this mission analysis module, we perform two DOC minimization cases of a 100-passenger regional jet aircraft. In the first case, we optimize mission profile parameters with a fixed aircraft geometry, to obtain the mission profiles to be used in the aerostructural optimization. In the second case, we perform an aerostructural optimization to obtain the optimized aircraft geometry with fixed mission profiles, using those obtained in the first optimization case. 
The paper is organized as follows. In Section II, we describe the solution methods we use for our mission analysis module. The descriptions of the aircraft configuration, engine model, and aerostructural solver are presented in Section III. Section IV presents the optimization problem formulations. We then present and discuss the results in Section V, and close with conclusion and future work in Section VI.

\section{Mission analysis methods}

In this section, we discuss the methods used to compute the mission characteristics, including fuel weight $W_{\text {fuel }}$, range $R$, and mission time $t$. Each of these characteristics can be computed by numerically integrating a given mission profile. We need to provide the mission profile parameters, such as altitude and Mach number for cruise segments, flight speed (Mach number or the knots indicated airspeed, KIAS), initial and final altitudes for climb and descent segments. The mission analysis also requires the initial takeoff weight and the final zero fuel weight (ZFW), which are determined by the weights and balance model in this work, for each mission. From this information, we then solve iteratively for the weight, range and time spent in each of the mission segments. Once the overall system solution is obtained, we can determine the characteristics for the entire mission.

In previous work on mission analysis, such as that of Henderson et al. [19], the mission is analyzed in a sequential fashion, analysing each segment in succession. In such a case, the previous segment's end weight is used as the starting point for the current segment, requiring that the segments be analyzed in sequence. In the current work, we have employed an all-at-once approach to solving the mission. The mission is broken up into several segments. Each segment is limited to one phase of flight, be that takeoff, climb, cruise, descent, or landing. By defining the various endpoint weights as states of the system, we are able to formulate a set of residual equations for each segment and assemble a set of nonlinear system residuals, $\mathcal{R}$, that is set to zero to solve the mission. This formulation of the mission allows each of the segments to be analyzed independently, based only on the states of the system.

\section{A. Mission segments}

In order to understand the formulation of the residual equations, we must first develop the equations for each of the segment types. In this work we have equations for climb, cruise, and descent. Startup, taxi, takeoff, and landing are handled through fuel fractions. The equations of numerical integration for each of these segment types is described below.

\section{Startup, taxi, takeoff, and landing}

The amount of fuel burn during startup, taxi, takeoff, and landing are computed using the fuel fraction method, where a constant ratio is used to define the weight at the end of the segment, given the initial weight for that particular segment. This method is expressed as

$$
W_{f}=(1-\zeta) W_{i},
$$

where $W_{i}$ and $W_{f}$ refer to the segment's initial and final weight, respectively. The fuel fraction values, $\zeta$, used in this work are listed in Table 1, following those suggested by Roskam [13], Raymer [23], and Sadraey [24].

Table 1: Fuel fraction values

\begin{tabular}{lccccc}
\hline Segment & Startup & Taxi & Takeoff & Landing & Taxi \& Shutdown \\
\hline Fuel fraction, $\zeta$ & 0.01 & 0.01 & 0.005 & 0.003 & 0.008 \\
\hline
\end{tabular}

\section{Cruise, climb and descent segments}

The fuel burn computation for the climb, cruise, and descent segments are derived from the range equation. To compute fuel burn, we first define the thrust specific fuel consumption, $c_{T}$, as:

$$
c_{T}=\frac{\text { Weight of fuel burned per unit time }(N / s)}{\text { Unit thrust }(N)} .
$$

We use an engine model to estimate the TSFC value, which is a property of the aircraft engine. The engine model used in this work is described in Section III. E. The rate of reduction of aircraft weight can thus be computed as,

$$
\frac{d W}{d t}=-c_{T} T
$$

where $W$ and $T$ denote aircraft weight and thrust, respectively. 
For the cruise segment, the equation for range, $R$, can be expressed as a definite integral of flight speed, $V$, over a certain time interval,

$$
R=\int_{t_{i}}^{t_{f}} V d t
$$

Substituting (3) to (4) lets us define the flight range given the specific range, i.e., the range per unit weight of fuel $\left(-V / c_{T} T\right)$, using the following expression,

$$
R=\int_{W_{i}}^{W_{f}}-\frac{V}{c_{T} T} d W
$$

where $W_{i}$ and $W_{f}$ refer to the weights at the start and the end of a cruise segment, respectively. Similarly, by rearranging (3), the cruise time can be computed as,

$$
t=\int_{W_{i}}^{W_{f}}-\frac{1}{c_{T} T} d W
$$

The range equation for the climb and descent segments are derived similarly. We use the following relation between flight speed and rate of climb, $R C$,

$$
R C=\frac{d h}{d t}=V \sin \gamma,
$$

where $h$ and $\gamma$ denote flight altitude and flight path angle, respectively. We approximate $R C$ based on the equation of motion given below,

$$
T_{\mathrm{av}} \cos \left(\phi_{T}+\alpha\right)-D-W \sin \gamma=\frac{W}{g} \frac{d V}{d t},
$$

where $T_{\text {av }}$ denotes the available thrust, $D$ denotes drag, and $g$ is the gravitational acceleration. The thrust inclination angle is denoted by $\phi_{T}$ (typicaly assumed to be zero [25]), and $\alpha$ refers to the angle of attack. With small angle approximations, we get the simplified equation as shown below,

$$
T_{\text {av }}-D-W \gamma=\frac{W}{g} \frac{d V}{d t}
$$

Similarly, the rate of climb can be approximated as $R C \approx V \gamma$. Rearranging and substituting (9) into this approximation gives us:

$$
R C=\frac{\left(T_{\mathrm{av}}-D\right) V}{W\left(1+\frac{V}{g} \frac{d V}{d h}\right)}
$$

For each point in the numerical integration we solve for a value of $\gamma$ that is consistent with the differential between the thrust and the drag to determine the actual rate of climb $R C$. Returning to (7), we can see that the time to climb is simply,

$$
t=\int_{h_{i}}^{h_{f}} \frac{1}{R C} d h
$$

where $h_{i}$ and $h_{f}$ are the initial and final altitudes for the climb segment. Note that for climb and descent we integrate over altitude, since this is the independently specified variable for these segments.

Substituting (3) into (7), we can obtain an expression for the fuel burn with respect to altitude,

$$
W_{\text {fuel }}=\int_{h_{i}}^{h_{f}}-\frac{c_{T} T}{R C} d h
$$

finally if we modify (4) to account for the climb angle so that,

$$
\frac{d R}{d t}=V \cos \gamma
$$

and substitute this equation into (7), we get the following expression for climb range,

$$
R=\int_{h_{i}}^{h_{f}} \frac{V \cos \gamma}{R C} d h .
$$

$$
4 \text { of } 24
$$


In this mission analysis module, the integrations to find the mission range, time, and fuel are solved numerically, by dividing each segment to a number of intervals.

Referring back to (10) we note that there is an undefined derivative $d V / d h$. This leads to three separate climb formulations, one for accelerated climb, one for constant velocity climb, and one for constant Mach climb. The constant velocity refers to the constant indicated airspeed (IAS) (the airspeed when the aircraft is at sea level under International Standard Atmospheric conditions) and it needs to be converted into the true airspeed (TAS) before we evaluate the term $d V / d h$. For the accelerated and constant velocity climb segments, we use (15) to compute $d V / d h$, where the second term goes to zero for the constant velocity case.

$$
\frac{d V}{d h}=\frac{\partial V_{\mathrm{TAS}}}{\partial h}+\frac{\partial V_{\mathrm{TAS}}}{\partial V_{\mathrm{IAS}}} \frac{\partial V_{\mathrm{IAS}}}{\partial h} .
$$

For constant Mach climb,

$$
\frac{d V}{d h}=M \frac{d a}{d h},
$$

where $a$ is the speed of sound. We compute the derivative terms $\partial V_{\mathrm{TAS}} / \partial h, \partial V_{\mathrm{TAS}} / \partial V_{\mathrm{IAS}}$, and $d a / d h$, using finite differencing.

\section{B. Residual equations}

Having defined the equations of numerical integration for the various segments, we now need to develop the residual equations for the nonlinear system. We have defined the states as the endpoint weights of each segment. This causes duplicate values of the weight at each node of the mission. Therefore the primary residual equations are that the end point weights of two adjacent segments must be equal,

$$
W_{f_{j}}-W_{i_{j+1}}=0,
$$

where $j=1, \ldots, N_{\text {seg }}$ denotes the segment index. The boundary conditions for the problem are formulated in a similar manner, i.e. that the initial weight of the first segment match the initial weight $W_{\text {TO }}$ and that the final weight of the last segment match the final weight $W_{\mathrm{ZF}}$, giving:

$$
W_{i_{1}}-W_{\mathrm{TO}}=0
$$

and

$$
W_{f_{N_{\text {seg }}}}-W_{\mathrm{ZF}}=0 .
$$

For the climb and descent segments, this single residual equations is sufficient as each segment adds only a single degree of freedom to the system ( $W_{i}$ is independent, $W_{i+1}$ is determined by the integration). However, for each cruise segment, both endpoints are state variables, therefore we need a second residual equation for each of these segments. For this second equation, we constrain the ratio of the various cruise segments to be fixed. In this case we constrain that all cruise segments be equal, but other ratios are possible. To implement this constraint, the range of the first cruise segment is calculated and used as a reference range, then for each other cruise segment we have the equation:

$$
\left(R_{\text {ref }}-R_{i}\right) / R_{\text {ref }}=0 .
$$

Note that we have scaled this residual by the reference range to improve the conditioning of the Jacobian of the system.

We then use a line search stabilized Newton's method to solve the nonlinear system, where the Jacobian for the Newton iteration is formed using finite differencing. This forces the weights of the various segments to be consistent with each other, providing a valid, continuous mission profile.

\section{Aerodynamic data}

To facilitate the efficient numerical integration of the individual flight segments, we use a surrogate model to approximate the required aerodynamic coefficients, $C_{L}, C_{D}$, and $C_{m}$. To generate the model, we sample a small number of points (we use 28 in this work) in the four dimensional space of Mach, altitude, angle of attack, and tail rotation angle, and compute the aerodynamic coefficients at these points. To avoid having ill-conditioned kriging equations, these design variables are normalized to $[0,1]$ intervals, before the kriging models are constructed and later on used. From this data, three surrogate models are generated, one for each coefficient. In our case, all of the moment coefficients are computed about a fixed reference point. Therefore we account for the variation in center of gravity location for the different mission segments using the following equation,

$$
\Delta M=W \cdot g \cdot\left(x_{\mathrm{cg}}-x_{\mathrm{ref}}\right),
$$


where $x_{\text {ref }}$ refers to the location of reference point used to compute pitch moment. This leads to a correction on the pitching moment coefficient,

$$
\Delta C_{m}=\frac{\Delta M}{\frac{1}{2} \rho S_{\mathrm{ref}} V^{2} \cdot \mathrm{MAC}},
$$

where $\rho, S_{\text {ref }}$, and MAC denote the atmospheric density, reference wing area, and the mean aerodynamic chord of the wing, respectively. The velocity $V$ used in this equation refers to the true airspeed.

To compute the drag at each point in the mission, we use a Newton method to search for the angle of attack $(\alpha)$ and tail rotation angle $(\eta)$ that satisfy lift and trim conditions simultaneously. The general equation for Newton's search algorithm is

$$
\mathbf{x}^{(k)}=\mathbf{x}^{(k-1)}-\mathbf{J}^{-1} \mathbf{f}\left(\mathbf{x}^{(k)}\right),
$$

where $k$ is the iteration index. The function $\mathbf{f}$ is to be driven to zero and $\mathbf{J}$ is the corresponding Jacobian. In this search procedure, the values for $\mathbf{x}, \mathbf{f}$, and $\mathbf{J}$ are

$$
\mathbf{x}=\left[\begin{array}{l}
\alpha \\
\eta
\end{array}\right], \quad \mathbf{f}=\left[\begin{array}{l}
C_{L}-C_{L_{\text {target }}} \\
C_{m}+\Delta C_{m}
\end{array}\right], \quad \mathbf{J}=\left[\begin{array}{cc}
\frac{\partial C_{L}}{\partial \alpha} & \frac{\partial C_{L}}{\partial \eta} \\
\frac{\partial C_{m}}{\partial \alpha} & \frac{\partial C_{m}}{\partial \eta}
\end{array}\right]
$$

Once the values of $\alpha$ and $\eta$ are determined for a given Mach and altitude, the drag surrogate model can be evaluated to determine the drag for that point. This drag computation is conducted at every point of numerical integration for each segment. Thus, the number of evaluations required is the product of the number of missions, the number of Newton iterations required to solve each mission system, the number of segments per mission, the number of intervals per segment, and the number of Newton iterations to compute the drag for each point. This can easily lead to thousands of potential evaluations per mission, which would be extremely expensive to compute directly. However, by using surrogate models for the aerodynamic models, the cost of this evaluation can be reduce to mere seconds.

\section{Kriging models}

The kriging approximation technique is a statistical interpolation method that is constructed by minimizing the mean squared error of the approximation, subjected to the unbiasedness constraint [26, 27]. We refer the reader to previous work for further details on the derivation and formulation of kriging models [26, 28, 29]. To build a kriging surrogate model, we need $N_{s}$ sample points, $\mathbf{x}$, and function values evaluated at those sample points, $\mathbf{y}_{s}$. A kriging predictor, $\hat{y}$, at an untried point $\mathbf{x}_{0}$ can then be expressed as,

$$
\hat{y}\left(\mathbf{x}_{0}\right)=\mathbf{F}\left(\mathbf{x}_{0}\right) \boldsymbol{\beta}+\mathbf{r}\left(\mathbf{x}_{s}, \mathbf{x}_{0}\right)^{T} \mathbf{R}^{-1}\left(\mathbf{y}_{s}-\mathbf{F}\left(\mathbf{x}_{s}\right) \boldsymbol{\beta}\right)
$$

where $\mathbf{R}$ denotes the correlation matrix of size $N_{s} \times N_{s}, \mathbf{F}(\mathbf{x})$ is a matrix of basis functions for the global model (whose entry values might depend on the location of $\mathbf{x}_{0}$ ), and $\boldsymbol{\beta}$ denotes the corresponding basis function coefficients. $\mathbf{r}_{0}\left(\mathbf{x}_{s}, \mathbf{x}_{0}\right)$ denotes the correlation vector between $\mathbf{x}_{0}$ and sample points. The first term of (25) is a global model representing the "function mean", and the second term is a zero-mean stochastic realization with variance $\sigma^{2}$ and non-zero covariance:

$$
\operatorname{Cov}\left[y\left(\mathbf{x}^{i}\right), y\left(\mathbf{x}^{j}\right)\right]=\sigma^{2} \mathbf{R}\left[R\left(\mathbf{x}^{i}, \mathbf{x}^{j}\right)\right],
$$

where $R\left(\mathbf{x}^{i}, \mathbf{x}^{j}\right)$ is a correlation function between any two sample points $\mathbf{x}^{i}$ and $\mathbf{x}^{j}$. The stochastic term represents localized deviations from the global model, which allows kriging models to perform interpolation between sampled data points.

For the global model, a low-order polynomial (similar to the polynomial model in a response surface), is commonly used. Typically, a constant global model is deemed sufficient and thus used in the kriging approximation [26, 30]. In which case, all entries in $\mathbf{F}\left(\mathbf{x}_{s}\right)$ are a scalar 1 and its coefficient vector, $\boldsymbol{\beta}$, is reduced to a scalar value $\beta$. Such a kriging model is typically referred to as ordinary kriging. In a "kriging with a trend" model, on the other hand, the global model is modeled by an analytical expression, which takes different values in space, to be the trend component [31].

The stochastic term is modeled as a Gaussian process, where the Gaussian exponential correlation is used for the spatial correlation function. Other correlation functions, such as exponential and cubic spline functions, can also be used. The formulation for the Gaussian correlation function is shown below,

$$
R\left(\mathbf{x}^{i}, \mathbf{x}^{j}\right)=\exp \left[-\sum_{k=1}^{N_{\mathrm{dv}}} \theta_{k}\left|x_{k}^{i}-x_{k}^{j}\right|^{2}\right],
$$

where $N_{\mathrm{dv}}$ is the problem dimension, $\theta_{k}$ is the unknown correlation parameter associated with the $k$-th design variable used to fit the model, and $x_{k}^{i}$ and $x_{k}^{j}$ are the $k$-th components of sample points $\mathbf{x}^{i}$ and $\mathbf{x}^{j}$. This property of the 
correlation function ensures that the approximation error of kriging is zero at sample points and increases with distance. A vector of correlation parameters (denoted as $\boldsymbol{\theta}$ ) determines the "strength" of correlation in each direction. These kriging hyperparameters are also referred to as distance weights. Large distance weight values correspond to weak spatial correlation, whereas small values correspond to strong spatial correlation [32]. These kriging hyperparameters are found via a maximum likelihood estimation (MLE) optimization, with the assumption that the approximation errors are normally distributed and that the process variance is stationary, i.e., it is independent of the locations in the design space. The gradient-free Hooke-Jeeves pattern search algorithm $[33,34]$ is used to solve the MLE optimization problem, as suggested by Lophaven et al. [35], which was also used in other previous work [28, 36, 37].

In this work, the kriging models are used to approximate the aerodynamic force and moment coefficients across the entire range of flight conditions in a flight mission, i.e. from takeoff to landing. We thus need to ensure that the design space of the kriging models cover the operating points of the entire mission. The range of values for the four kriging variables are shown in Table 2.

Table 2: Flight condition value ranges

\begin{tabular}{lcc}
\hline Kriging variables & Lower bound & Upper bound \\
\hline Mach & 0.2 & 0.9 \\
Altitude & $0 \mathrm{ft}$ & $45000 \mathrm{ft}$ \\
Angle of attack $(\alpha)$ & $-5.0^{\circ}$ & $12.0^{\circ}$ \\
Tail rotation angle $(\eta)$ & $-10.0^{\circ}$ & $12.0^{\circ}$ \\
\hline
\end{tabular}

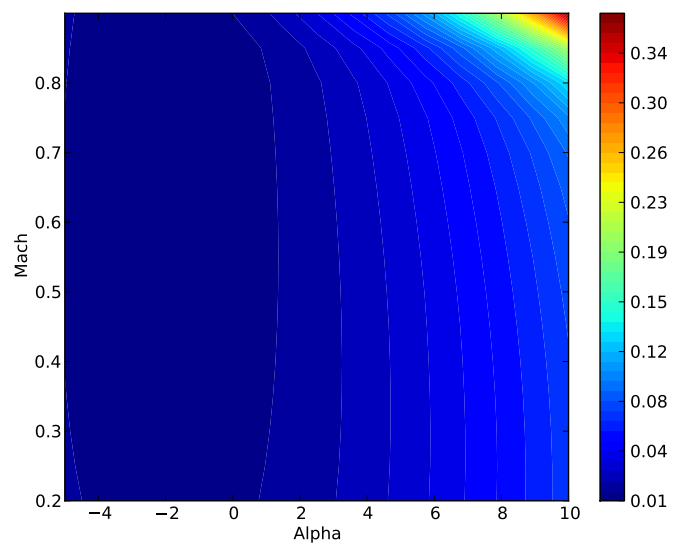

Figure 1: $C_{D}$ distribution at $35000 \mathrm{ft}$ and tail rotation angle $0^{\circ}$ (for pressure drag).

Figure 1 shows the $C_{D}$ distribution (for pressure drag only) of a regional jet, at $35000 \mathrm{ft}$ altitude and tail angle of $0^{\circ}$. These $C_{D}$ values are obtained from a medium-fidelity aerodynamic solver, TriPan, which uses the panel method. From this $C_{D}$ distribution, we observe a high drag gradient, especially in the high Mach number and high angle of attack region. Ordinary kriging model is unable to capture this high drag gradient well, as shown in Figure 2a. In general, accuracy can be improved by increasing the number of samples in the kriging model construction, but doing so will significantly increase the computational cost and time, which defeats the purpose of using surrogate models in the first place. Instead, we introduce a trend in the kriging model by using a nonconstant global model for $C_{D}$ kriging approximation. The basis functions are selected to follow the trend of $C_{D}$, especially in the high drag gradient region (high Mach, high $\alpha$ ). We thus select the following equation as the global model for $C_{D}$ kriging approximation,

$$
f(\mathbf{x})=\beta_{0}+\phi(M, \alpha) \cdot \beta_{1} .
$$

Here, $\mathbf{x}$ is a vector of $[M, h, \alpha, \eta]$, where $M, h, \alpha$, and $\eta$ refer to Mach number, altitude, angle of attack, and tail rotation angle, respectively. The function $\phi(M, \alpha)$ is given below,

$$
\phi(M, \alpha)=\left\{\begin{array}{lll}
\frac{1}{1-M^{2}} & \text { if } \quad \alpha \leq 1.0 \\
\frac{\alpha^{2}}{1-M^{2}} & \text { if } \quad \alpha \geq 1.0
\end{array} .\right.
$$

The constant numerator for $\alpha \leq 1.0$ is used to remove the quadratic profile (with respect to $\alpha$ ) in low $\alpha$ region, to be consistent with the $C_{D}$ distribution obtained from the aerodynamic solver. The basis function vector, $\mathbf{F}(\mathbf{x})$, and the 
coefficient vector, $\boldsymbol{\beta}$, are thus expressed as follows,

$$
\begin{gathered}
\mathbf{F}(\mathbf{x})=[1, \phi(M, \alpha)] \\
\boldsymbol{\beta}=\left[\beta_{0}, \beta_{1}\right]^{T} .
\end{gathered}
$$

Thus at an evaluation point $\mathbf{x}_{0}=\left[M_{0}, h_{0}, \alpha_{0}, \eta_{0}\right]$, the kriging equation can be expressed as

$$
\hat{y}\left(\mathbf{x}_{0}\right)=\beta_{0}+\beta_{1} \phi\left(M_{0}, \alpha_{0}\right)+\mathbf{r}\left(\mathbf{x}_{s}, \mathbf{x}_{0}\right)^{T} \mathbf{R}^{-1}\left[\mathbf{y}_{s}-\beta_{0}-\beta_{1} \phi\left(M_{0}, \alpha_{0}\right)\right] .
$$

The kriging model construction procedure will determine the basis function coefficients, $\beta_{0}$ and $\beta_{1}$, by the mean square error minimization, subject to the unbiasedness constraint.

The comparison between ordinary kriging and kriging with specified basis functions (trend) for $C_{D}$ approximation is shown in Figure 2. From these figures, we can observe that the basis functions we specify for the kriging global model significantly improve the accuracy of kriging models in the high drag gradient area. This accuracy improvement will in turn improve the accuracy of mission analysis with the same number of samples. The implementation of kriging with specified basis functions only requires an additional $0.01 \mathrm{~s}$ in the construction time, from the $0.2 \mathrm{~s}$ required to construct an ordinary kriging model. This additional computational time is considered very minimal.

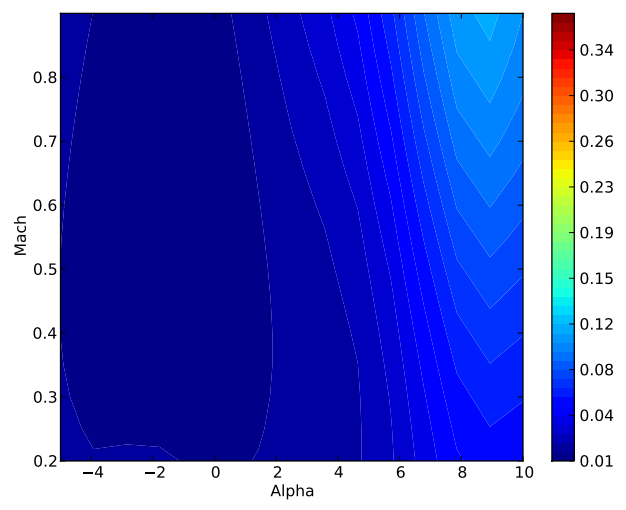

(a) Ordinary kriging

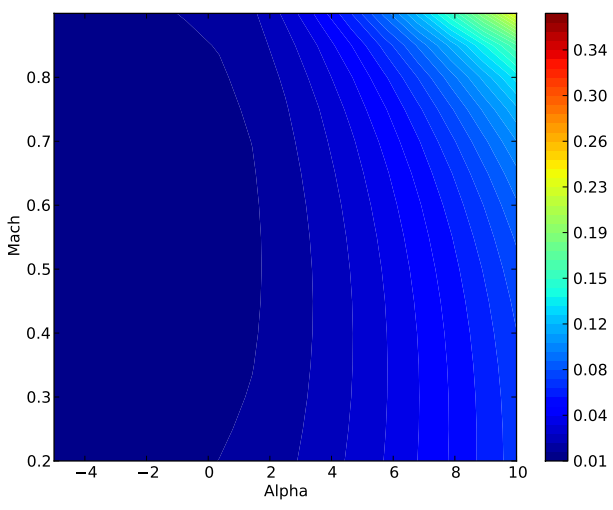

(b) Kriging with specified trend

Figure 2: Comparing $C_{D}$ approximations (at $35000 \mathrm{ft}$ and $0^{\circ}$ tail rotation angle) using ordinary kriging and kriging with specified trend surrogate models (for pressure drag).

For $C_{L}$ and $C_{M}$, the profiles do not exhibit a high gradient in the design space, thus ordinary kriging models are deemed sufficient to approximate these quantities. In this work, 28 samples are used to construct the kriging models.

\section{Optimization problem description}

In this section, we describe the aircraft configuration, as well as its wing structural model, that we use in the optimization problems. The coupled aerostructural solver is also described. We then briefly talk about the DOC computation, engine model, weight and balance components of the analysis. Finally we discuss the software architecture for the mission analysis module.

\section{A. Aircraft configuration}

The aircraft being considered is a 100-passenger regional jet. This aircraft is configured with low swept-back wings, two aft engines mounted on the fuselage, and a T-tail, similar to the other regional aircraft such as the Bombardier CRJ1000, McDonnell Douglas DC-9, and Fokker 100. The fuselage is designed to accommodate a five-abreast seating, which gives an overall fuselage length of $108.6 \mathrm{ft}$ with a width of $10.8 \mathrm{ft}$. The wing for this initial configuration is a winglet design, with a sweep of $30^{\circ}$ at the leading edge, giving a wing area of $989 \mathrm{ft}^{2}$ and a span of $84.9 \mathrm{ft}$. A 3-view of this configuration is shown in Figure 3.

\section{B. Wing structural model}

Figure 4 illustrates the three-dimensional wing-tail structure with the internal structure layout that we use in this work. The thickness and pressure distributions are also shown. A 3D wing and tail finite element structural geometry is constructed using shell elements. The wing box structure consists of a front spar at $15 \%$ chord, rear spar at $65 \%$ chord, and a subspar to support the landing gear structure. The thickness of this baseline wing is $14 \% t / c$ for the entire 

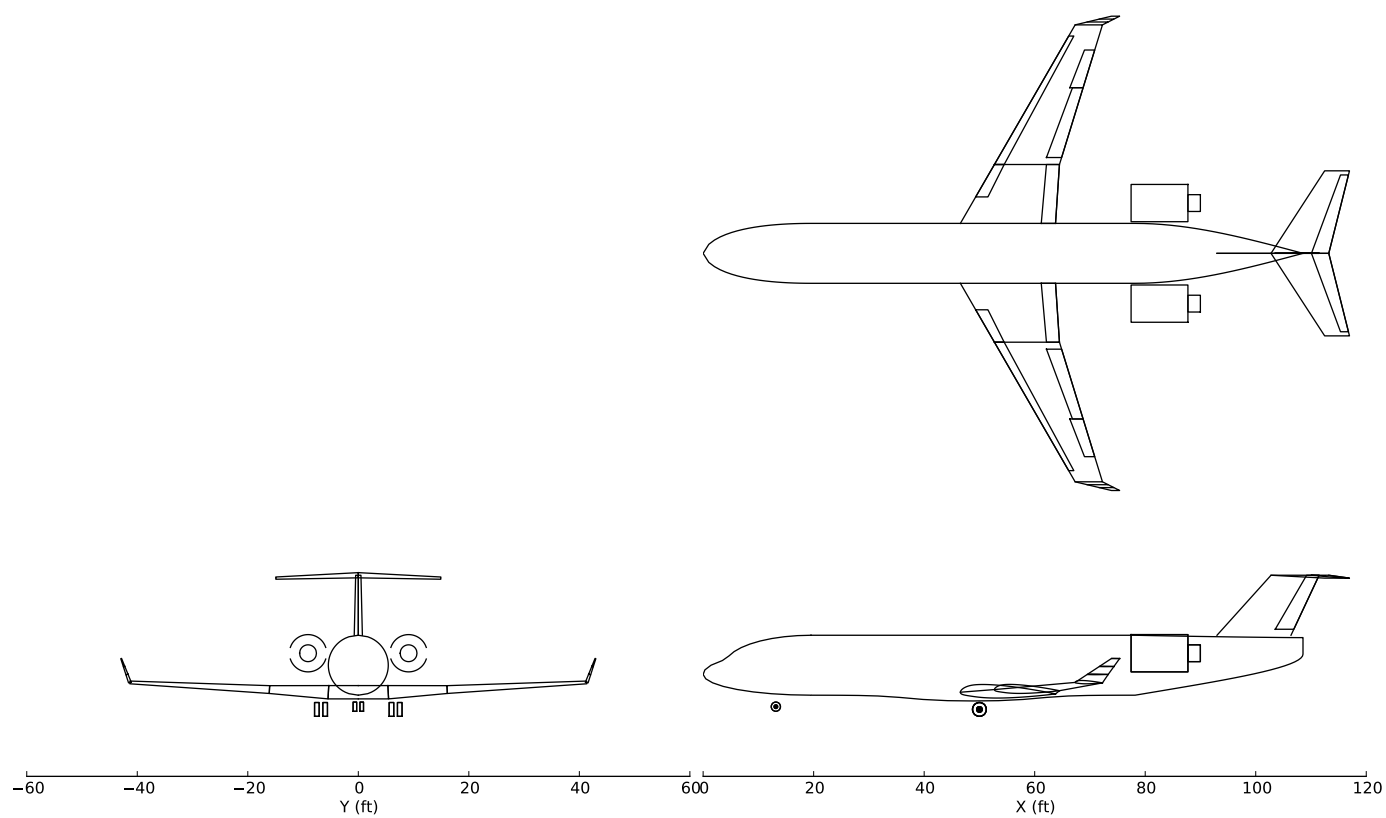

Figure 3: A 3-view drawing of the baseline 100-passenger regional jet.

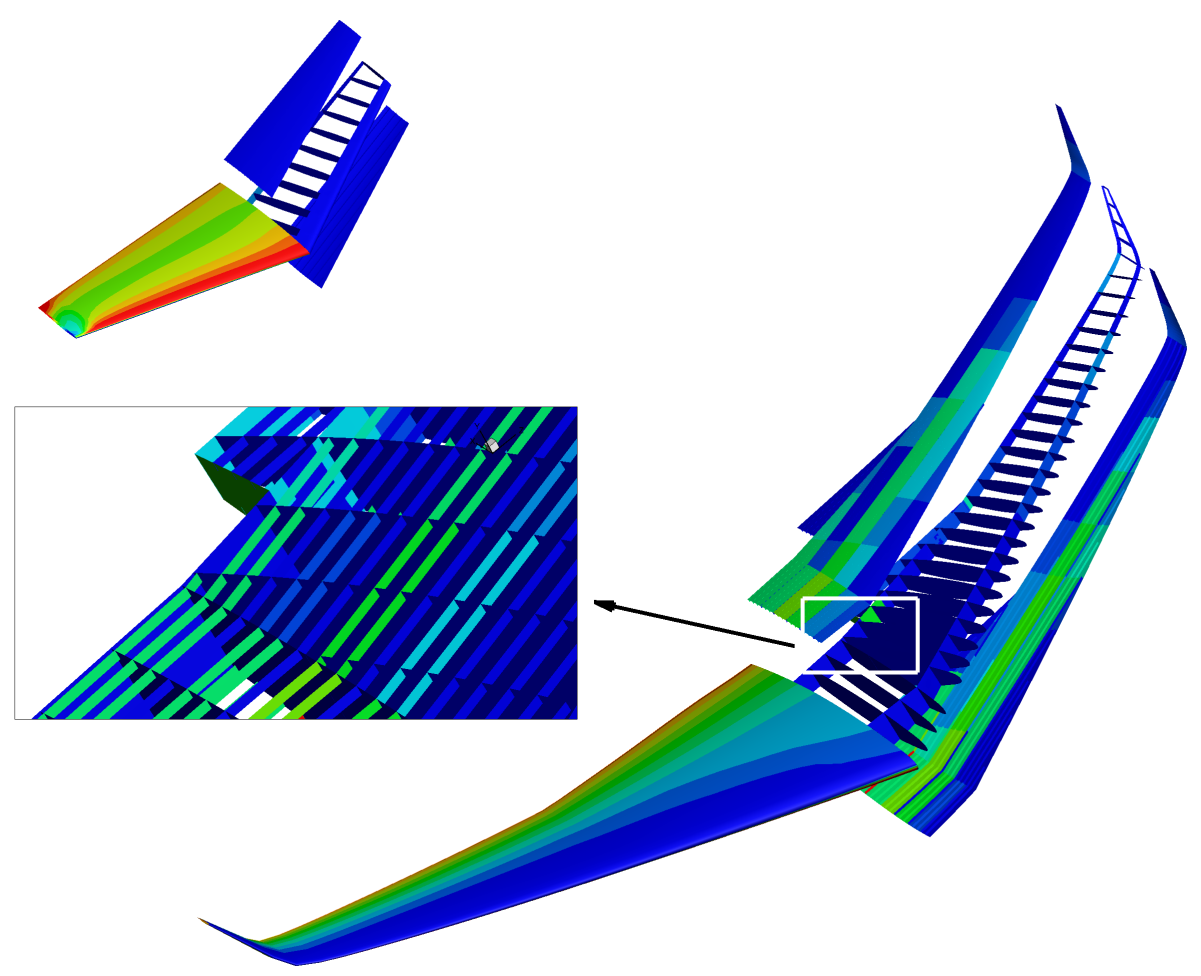

Figure 4: Thickness and pressure distributions of a representative wing-tail structure, showing the geometry and the internal structural layout. 
centerbody section, $10 \% t / c$ for the wingtip and winglet, and linearly varying in between. Structural components that are defined include ribs, spars, skins, and stringers, with the ribs being oriented flow-wise in the centerbody and winglet, and perpendicular to the rear spar in the main wing. A fixed leading edge structure is added for better load transfer between the aerodynamic forces and structure; however, this structure is not sized and not accounted for in the total weight. Top and bottom blade stringers are also included in the model, oriented perpendicularly to the ribs. Since buckling is not considered at the moment, the required stringer thicknesses would not be captured. Therefore, the stringer thickness are linked to the skin thickness for a better weight prediction.

The minimum thickness for all components is set at $0.080 \mathrm{in}$, except for the ribs which is set at slightly higher, since they are not sized by buckling or the loading from the control and high-lift device attachments. Also, adjacency thickness constraints are enforced that limit skin thickness variations between neighbouring patches. Aluminium 7075 is used for the top skin and top stringer, and aluminium 2024 is used for the rest of the structure. The material properties for both of these alloys are summarized in Table 3. For stress constraint considerations, the limit is the lesser of the ultimate stress with a safety factor of 1.5 or the yield stress; the lower value for each material is highlighted in the table.

Table 3: Material properties [38] of aluminium 2024 and 7075, including fatigue data [39] at 60000 cycles.

\begin{tabular}{rccl}
\hline & 2024-T4 & 7075-T73 & \\
\hline$\rho$ & 0.100 & 0.102 & $\mathrm{lb} / \mathrm{in}^{3}$ \\
$E$ & 10600 & 10400 & $\mathrm{ksi}$ \\
$\nu$ & \multicolumn{2}{c}{0.330} & \\
$\sigma_{y}$ & 40.0 & 56.0 & $\mathrm{ksi}$ \\
$\sigma_{\text {ult }}$ & 62.0 & 67.0 & $\mathrm{ksi}$ \\
$\sigma_{\text {fat. }}$ & 12.25 & 8.4 & $\mathrm{ksi}$ \\
\hline
\end{tabular}

\section{Aerostructural solver}

The coupled aerostructural solution is obtained from a high-fidelity structural solver, TACS, and a medium-fidelity aerodynamic solver, TriPan. Both solvers are developed by Kennedy and Martins [40]. The Toolbox for the Analysis of Composite Structures (TACS) is a parallel finite-element solver purposely built for the analysis and optimization of composite structures. TACS is used to solve the wing-tail structure. TriPan calculates the aerodynamic forces and moments of inviscid, incompressible, external lifting flows on unstructured grid using surface pressure integration, with constant source and double singularity elements. The surface of the body is discretized with quadrilateral and triangular panels. TriPan also computes the aerodynamic loading at various flying shapes. This aerodynamic solver is also used to determine the lift, drag, and moment coefficients $\left(C_{L}, C_{D}\right.$, and $\left.C_{m}\right)$ used to construct the surrogate models for the mission analysis. Both the structural and aerodynamic solvers are equipped with an accurate and efficient analytic gradient computation routine, allowing a gradient-based optimizer to be used. Through the optimization, the optimum thickness distribution of each structural member is determined. The fluid-structure interface in the aerostructural solver is done via a load displacement transfer procedure, where rigid links are used to extrapolate the displacements from the structural surface to the outer-mold line (OML) of the aerodynamic surface [41, 42]. A coupled adjoint method that allows us to compute gradients of multidisciplinary functions of interest is one of the critical components that make high-fidelity aerostructural optimization tractable for large numbers of design variables [9, 43].

\section{DOC computation}

The cost estimates for each component in DOC computation are derived based on empirical methods [44]. The component breakdown, and the key contributing factors to each component, of the DOC computation are given in Table 4. From the mission analysis procedure, we obtain the mission range, time, total amount of fuel burned (fuel weight). MTOW is obtained from the weight and balance model. The technology year is used to compute the escalation factor in the cost computation to the current year. A fixed fuel price of $\$ 3 \mathrm{USD} /$ gal is used in the computation.

\section{E. Engine model}

The engine model is based on an engine map from data generated by Gas Turb [45], a commercially available gas turbine performance software commonly used within industry. Two sets of data are generated: (1) maximum climb, with data at various Mach and altitude, and (2) cruise, with data at various Mach, altitude, and thrust setting. For each set of engine data, three additional sets are generated for three different bypass ratios (BPR). These data allow the mission analysis to determine the available thrust and the associated specific fuel consumption at a particular flight condition, by performing a high-dimensional linear interpolation. 
Table 4: The dependency of DOC components on aircraft specifications and mission performance.

\begin{tabular}{lccccc}
\hline Components & MTOW & $\begin{array}{c}\text { Takeoff } \\
\text { Thrust }\end{array}$ & $\begin{array}{c}\text { Fuel } \\
\text { Weight }\end{array}$ & $\begin{array}{c}\text { Block } \\
\text { Time }\end{array}$ & Range \\
\hline Crew & $\checkmark$ & & & $\checkmark$ & \\
Attendent & & & & $\checkmark$ & \\
Fuel & & & $\checkmark$ & & \\
Oil & & & $\checkmark$ & & \\
Airport Fees & $\checkmark$ & & & & $\checkmark$ \\
Navigation Fees & $\checkmark$ & & & $\checkmark$ & \\
Airframe Maint. & $\checkmark$ & & & $\checkmark$ & \\
Engine Maint. & & $\checkmark$ & & $\checkmark$ & \\
Insurance & $\checkmark$ & & & $\checkmark$ & \\
Financing & $\checkmark$ & & & $\checkmark$ & \\
Depreciation & $\checkmark$ & $\checkmark$ & & $\checkmark$ & \\
Registry Taxes & $\checkmark$ & & & \\
\hline
\end{tabular}

\section{F. Weight and balance}

The total weight of the aircraft is decomposed into its various structural and system components, such as wing, fuselage, furnishings, payload, and fuel, each with their own CG location estimate, as well as a forward and an aft CG limit estimate. The addition of these component weights and moments gives an estimate of the entire aircraft's weight, and the nominal, forward, and aft CG locations. The nominal CG estimate can be used for the mission analysis during a cruise flight condition, while the CG limits can be used for the analysis of critical load cases. During the optimization, the weight and CG location of these components can be individually updated, giving a more accurate picture of the aircraft's weight and balance in the mission optimization as the fuel is decremented in the integration.

\section{G. Software architecture}

The main computational part of our mission analysis module is implemented in Fortran, and wrapped in Python. This combination has been proven to be very effective. Fortran offers a significantly faster computational time as compared to Python, and the object-oriented Python provides the more practical user interface (scripting), ease of use of a class object, and plotting features. Using Python at scripting level has also facilitated the integration of the different fortran modules (e.g., aerodynamic solver, mission analysis, kriging surrogate models, atmospheric module, and engine model). The sensitivity computation in the fortan modules is done using Tapenade [46], which performs algorithmic diffentiation (AD) directly on the modules in an integrated fashion. The optimization problem is solved using SNOPT [47], a gradient-based sequential quadratic programming optimizer, which is used within pyOpt, an object-oriented framework for nonlinear optimization [48]. The aerostructural optimization is performed on a massively parallel supercomputer [49]. In total, we use 96 processors to complete the aerostructural optimization, with the breakdown shown in Table 5 .

Table 5: Computational resources breakdown for the parallel aerostructural optimization.

\begin{tabular}{lcccc}
\hline \multirow{2}{*}{ Group } & $\begin{array}{l}\text { Number of } \\
\text { Load Cases }\end{array}$ & \multicolumn{3}{c}{ Number of Processors } \\
\cline { 3 - 5 } & & Aero & Struct & Total \\
\hline Maneuver & 4 & $6 \times 4$ & $6 \times 4$ & 48 \\
Landing & 5 & & 10 & 10 \\
Stability & 2 & 8 & 8 & 16 \\
\hline Aerodynamics & 28 & 21 & & 21 \\
Mission Analysis & 2 & & & 1 \\
\hline Total & & & & 96 \\
\hline
\end{tabular}

\section{Optimization problem formulation}

In this section, we present the two DOC minimization cases performed in this work. The first is a mission profile optimization where the aircraft geometry and engine are assumed fixed, whereas the second one is an aerostructural 
optimization with fixed mission profiles. This mission profile, obtained from the first optimization case, is used for the aerostructural optimization.

\section{A. Mission profile optimization}

We demonstrate the use of the described mission analysis framework to minimize DOC by optimizing the mission profile. Two different missions are considered for this aircraft: a $2000 \mathrm{nmi}$ long range cruise (LRC) mission and a $500 \mathrm{nmi}$ typical range cruise (TRC) mission, both with 100 passengers. The LRC mission ensures the aircraft is capable of carrying the full fuel required for the maximum range, while the objective of the optimization is to minimize the DOC based on the TRC mission. The LRC mission also has an impact on the DOC, as its fuel weight contributes to the aircraft MTOW, which goes into the DOC computation.

The mission profile for both the TRC and LRC missions are illustrated in Figure 5 (not drawn to scale). A single cruise segment is assumed for the TRC mission, whereas the LRC mission is assumed to have two cruise segments. The entire cruise segment for the LRC mission, from the initial cruise, through the step climb, to the final cruise, is done at a constant Mach number, with a single Mach number design variable. From $10000 \mathrm{ft}$, the climb is done at a constant knots indicated airspeed (KIAS), until it intercepts the desired cruise Mach number, at which point the climb is done at a constant Mach number. The descent is also done in a similar fashion, with a constant Mach descent followed by a constant KIAS descent. This procedure is implemented to reflect common operational procedures.

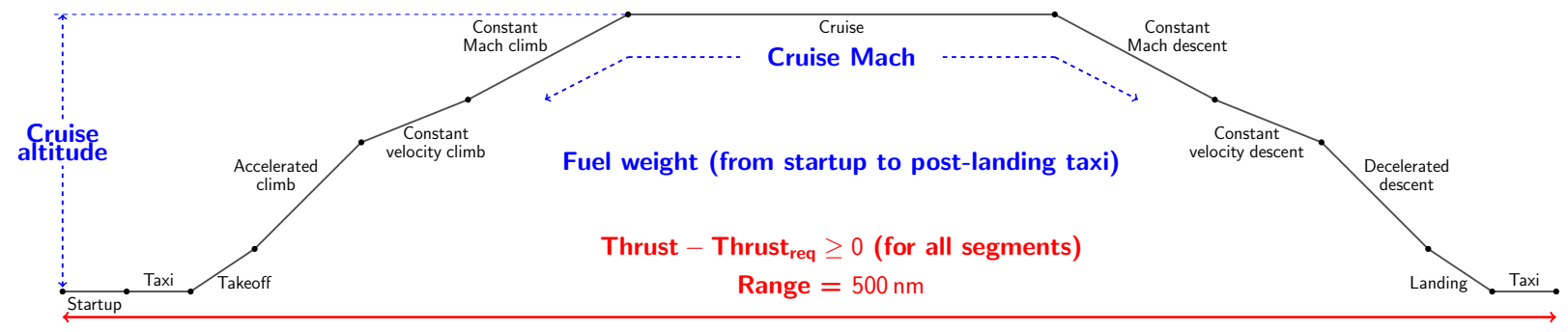

(a) $500 \mathrm{nmi}$ mission

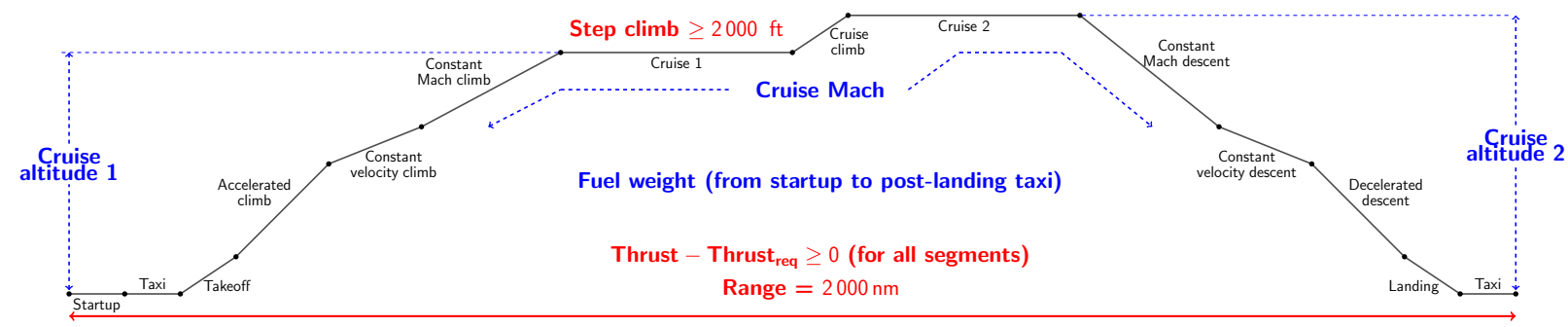

(b) 2000 nmi mission

Figure 5: Mission profile for the TRC and LRC missions (not drawn to scale).

The optimization problem formulation is presented in (33) below. The design variables and constraints are also shown in Figure 5 (indicated in blue and red, respectively). Note that the altitudes shown here, denoted as $h$, refer to the cruise altitude. The subscripts 1 and 2 in $h_{\mathrm{LRC}}$ denote the first and second cruise segment in the LRC mission, respectively.

$$
\begin{aligned}
\text { minimize } & \text { DOC } \\
\text { w.r.t } & 2 \text { fuel variables }: W_{\text {fuel }_{\text {TRC }}}, W_{\text {fuel }_{\text {LRC }}} \\
& 2 \text { cruise Mach numbers }: M_{\mathrm{TRC}}, M_{\mathrm{LRC}} \\
& 3 \text { cruise altitudes }: h_{\mathrm{TRC}}, h_{\mathrm{LRC}_{1}}, h_{\mathrm{LRC}_{2}} \\
\text { such that } & R_{\mathrm{TRC}}=500 \mathrm{nmi} \\
& R_{\mathrm{LRC}}=2000 \mathrm{nmi} \\
& \text { Thrust }- \text { Thrust }_{\text {req }} \geq 0.0 \\
& h_{\mathrm{LRC}_{2}}-h_{\mathrm{LRC}_{1}} \geq 2000 \mathrm{ft}
\end{aligned}
$$

The first two constraints are imposed to ensure that the mission profile can fly the range requirements for the two missions. The thrust requirement constraint is imposed to all segment intervals (for the numerical integration computation), and they all have to be positive. Lastly, the step climb altitude for the LRC mission has to be larger than 
$2000 \mathrm{ft}$, to account for the traffic separation rule for aircraft operating under IFR, as specified in FAR §91.179 [50]. The aircraft geometry and engine properties are fixed for this optimization. The maximum thrust of the engine is set at $14000 \mathrm{lbf}$, and the bypass ratio (BPR) is set at 11.5.

\section{B. Aerostructural optimization problem}

For the coupled aerostructural optimization, we consider the same $2000 \mathrm{nmi}$ LRC mission and a $500 \mathrm{nmi}$ TRC mission, both with 100 passengers and fuel for a $100 \mathrm{nmi}$ reserve. The DOC model computes the cost based on mission characteristics such as total flight time, total fuel burn, and cruise Mach number, as well as aircraft parameters such as MTOW.

Figure 6 shows the XDSM (eXtended Design Structure Matrix) diagram [51] of the aerostructural optimization problem. The black and shaded grey lines in the XDSM diagram represent process and data flows, respectively. The design variables for this optimization, $\mathbf{x}$, include the global variables, $\mathbf{x}_{\text {global }}$, aerodynamic variables, $\mathbf{x}_{\text {aero }}$, structural

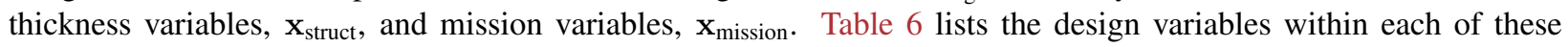
groups, as well as the state variables $\left(\mathbf{y}_{\text {aero }}\right.$ and $\mathbf{y}_{\text {struct }}$ ). At each optimization iteration, a multidisciplinary analysis (MDA) performs coupled aerostructural analyses and evaluate some of the optimization constraints, i.e. thicknesses, stresses, and static margin for stability. To build kriging models (in the surrogate model module), we need aerodynamic samples. These samples are evaluated in the aerodynamic module at 28 fixed sample locations. Once the kriging models are built, they are then passed to the last module, which performs mission analysis at fixed mission profiles.

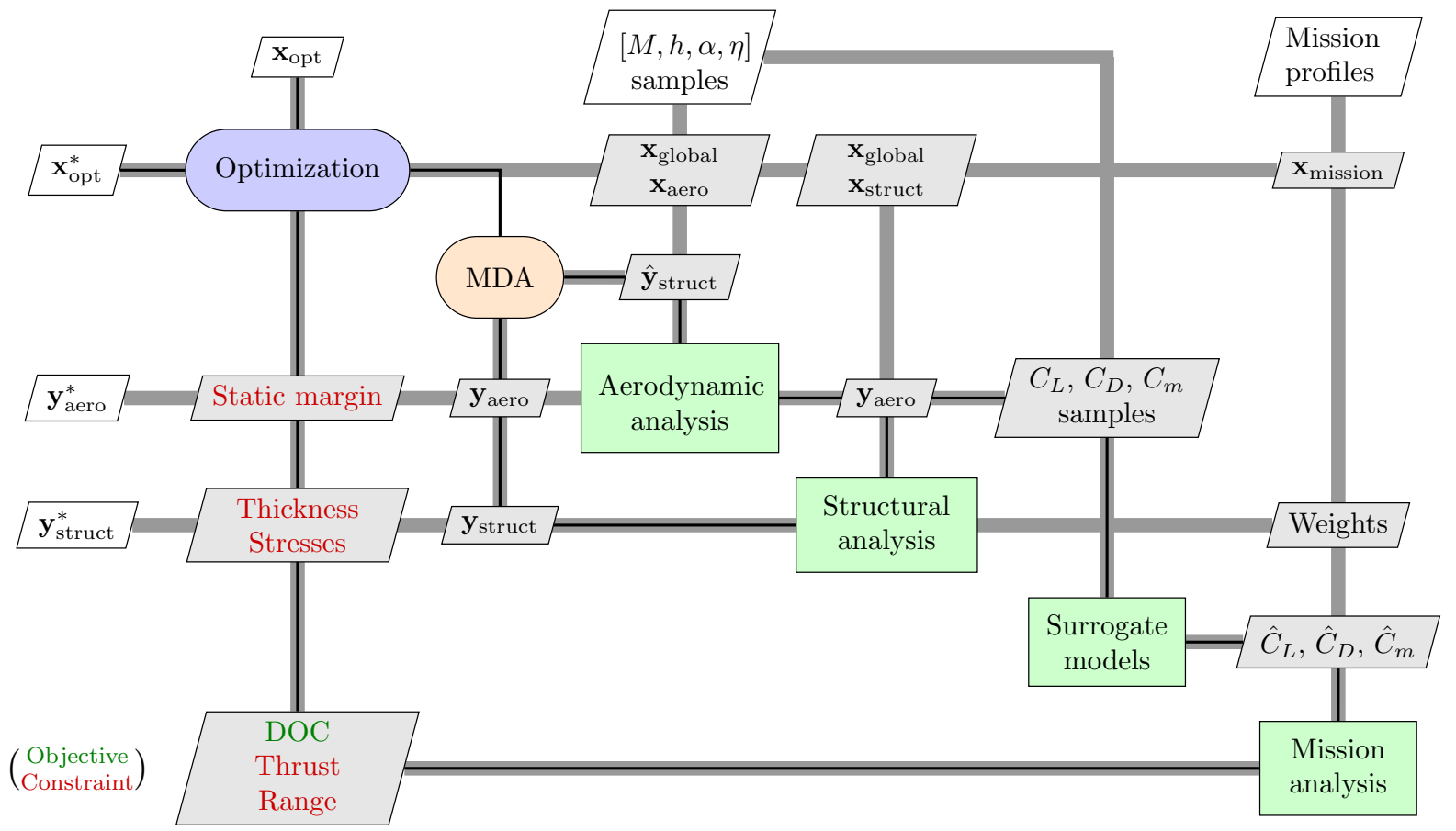

Figure 6: The XDSM diagram of the aerostructural optimization with surrogate-based mission analysis.

Table 6: List of design variables and state variables for the aerostructural optimization

\begin{tabular}{ll}
\hline \multicolumn{1}{c}{$\mathbf{x}$} & \multicolumn{1}{c}{ Design variables } \\
\hline $\mathbf{x}_{\text {global }}$ & Wing twists \\
$\mathbf{x}_{\text {aero }}$ & Angles of attack, tail angles \\
$\mathbf{x}_{\text {struct }}$ & Structural thicknesses \\
$\mathbf{x}_{\text {mission }}$ & Fuel weights for TRC and LRC missions \\
\hline \multicolumn{1}{c}{$\mathbf{y}$} & \multicolumn{1}{c}{ State variables } \\
\hline $\mathbf{y}_{\text {aero }}$ & Pressure coefficients \\
$\mathbf{y}_{\text {struct }}$ & Displacements and weights \\
\hline
\end{tabular}


In addition to the coupled aerodynamic loading obtained from TriPan, other loads are also considered, including load from self-weight, secondary weight from control surfaces and high-lift devices that are not part of the structural model, as well as fuel weight loads. The inertia load is based on the weight of the structure at the corresponding load condition. In addition, the secondary weights from the control surfaces and high-lift devices are also accounted for, determined based on the method by Torenbeek [52]. There are weight estimates for each of the leading edge slats, trailing edge flaps, ailerons, as well as the fixed portion of the leading edge and trailing edge. These weight estimates are functions of the aircraft's MTOW and the area of the device. These loads are applied to the leading edge spar and the rear-most spar at each rib bay. Fuel loading is also applied to the wing box, with the load applied on the bottom skin for a positive $g$ loading, while for a negative $g$ loading, the load is applied on the top skin only.

These loads are applied to several certification maneuver conditions, and the stresses from these flight conditions are constrained to be within the lesser of the material yield stress $\sigma_{y}$, or the ultimate stress $\sigma_{u l t}$ with a safety factor of 1.5. In total, four flying load cases with different loads being applied are considered: a pull up condition of $2.5 \mathrm{~g}$ at both MTOW and ZFW, and a pushover condition of $-1.0 \mathrm{~g}$ at MTOW. In addition, fatigue is also considered at MTOW by using a reduced allowable stress obtained from Hangartner [39], assuming a service life of 60000 cycles, with a safety factor of 1.3. The maneuver flight conditions and fatigue load case are analyzed at the optimized cruise Mach number and $70 \%$ of the cruising altitude. For all flight conditions, lift $(L)$ and moment $(m)$ are also constrained to ensure the wings are generating sufficient lift and that the aircraft is trimmed.

In addition to the maneuver load cases considered above, landing load cases are also considered to account for the forces transferred from the landing gear to the wingbox. Five landing conditions are applied at maximum landing weight (MLW) according to the requirements set out by FAR-25 [53], taking into consideration the level landing conditions (§25.479) and side load conditions (\$25.485). In order to determine the actual load that is to be applied to the wing box structure, the landing gear is modeled as a separate beam structure, assumed to be connected to the wing box structure at three points with the translational DOF constrained but not the rotational DOF. By applying the load at the axle, the reaction forces at the three attachments points are determined, and that reaction forces are applied to the actual wing box structure distributed over an area.

Aircraft stability is also enforced with two stability conditions. One with the aircraft at MTOW and one with the aircraft at ZFW. Both cases are simulated at the cruise altitude and speed with an aft center of gravity. The static margin $K_{n}$ of these two load cases are determined using

$$
K_{n}=-\frac{C_{M_{\alpha}}}{C_{L_{\alpha}}}
$$

and are constrained to be $K_{n} \geq 0.05$. The approximation of the static margin constraint is also described in more detail in previous work [54]. In total, five angle of attack variables, one for each flight conditions, are added to provide the required lift, and five tail angles are added to ensure the aircraft is trimmed. The shape and thickness design variables are illustrated in Figure 7. To summarize, the optimization formulation is as follows:

$$
\begin{aligned}
\text { minimize } & \text { DOC } \\
\text { w.r.t } & 278 \text { panel thicknesses }: t \\
& 2 \text { fuel variables }: W_{\text {fuel }} \text { TRC } \\
& 5 \text { angles of attack }: \alpha \\
& 5 \text { tail angles }: \eta \\
& 5 \text { wing twists }: \theta \\
\text { such that } & t_{i} \geq 0.080 \mathrm{in} \\
& R_{\text {TRC }}=500 \mathrm{nmi} \\
& R_{\mathrm{LRC}}=2000 \mathrm{nmi} \\
& \text { Thrust }- \text { Thrust } \\
& K_{n} \geq 5 \% \\
& \text { Additional constraints in Table } 7
\end{aligned}
$$

The first constraint defines the minimum gauge thickness bound of $0.080 \mathrm{in}$. The ranges as obtained from the mission analyses are also constrained to ensure that the mission requirements are satisfied. The thrust requirement constraint is imposed to all segment intervals, and they all have to be positive. The table also summarizes the additional constraints for each load case to ensure that the aircraft can generate enough lift, is trimmed with $m_{c g}=0$, is within allowable stresses, and has the correct fuel loading. The Kreisselmeier-Steinhauser (KS) constraint aggregation technique $[55,56]$ is used to combine the thousands of stress constraints into eight for each load case, four for each wing and tail. 
Table 7: Aerostructural specific constraints for each of the operating conditions considered.

\begin{tabular}{lcccccc}
\hline Group & Mach & Alt (ft) & $C_{m}=0$ & $K S(\sigma) \leq$ & Weight & Load Factor \\
\hline Maneuver & 0.65 & 25900 & fwd-CG & $\min \left(\sigma_{y}, \sigma_{u l t} / 1.5\right)$ & MTOW & 2.5 \\
& 0.65 & 25900 & fwd-CG & $\min \left(\sigma_{y}, \sigma_{u l t} / 1.5\right)$ & ZFW & 2.5 \\
& 0.65 & 25900 & fwd-CG & $\min \left(\sigma_{y}, \sigma_{u l t} / 1.5\right)$ & MTOW & -1.0 \\
& 0.65 & 25900 & fwd-CG & $\sigma_{\text {fatigue }}$ & MTOW & 1.3 \\
\hline Landing $(\times 5)$ & - & - & - & $\min \left(\sigma_{y}, \sigma_{u l t} / 1.5\right)$ & MLW & - \\
\hline Stability & 0.65 & 37000 & aft-CG & - & MTOW & 1.0 \\
& 0.65 & 37000 & aft-CG & - & ZFW & 1.0 \\
\hline
\end{tabular}

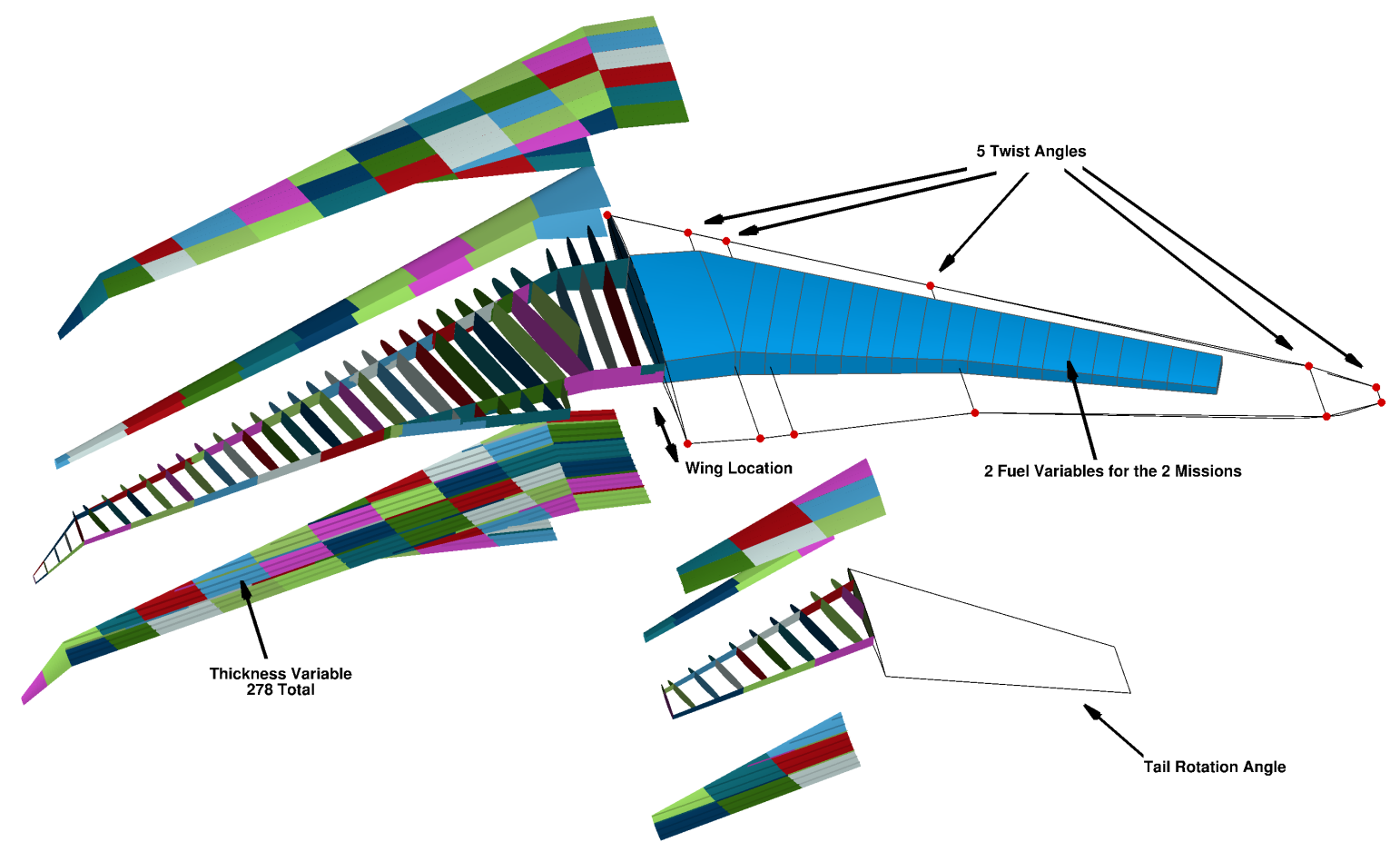

Figure 7: Design variables considered for the aerostructural optimization.

The structural mesh for the wing and tail consists of $94684,2^{\text {nd }}$ order elements, with a total of 542556 DOFs. The TriPan mesh consists of 30 spanwise panels and 15 chordwise panels. An inital, structural-only, weightminimization optimization is performed to determine the initial thickness distribution of the structural panels. For this optimization, the geometry is fixed, and the structure is loaded with a fixed aerodynamic load. The solution from this optimization is then used as the initial starting thickness distribution for the aerostructural optimizations.

\section{Optimization results}

The results from the mission profile optimization, as well as an aerostructural optimization that utilizes the optimized mission profile, are presented and discussed in this section. For both optimization cases, the numerical integrations within the mission analysis are done by dividing each segment into 50 intervals.

\section{A. Mission profile optimization results}

The results of the mission profile optimization (33) are presented here. The optimized mission profiles are presented in Table 8 and Table 9, respectively, and are illustrated in Figure 8. These figures display some mission segment characteristics, including the range, time, altitude, flight speed, and the amount of fuel burn. Note that "SL" refers to sea-level altitude. For the TRC mission, the aircraft flies at a relatively high Mach number of 0.81 for cruise, whereas for the LRC mission, it flies at a lower Mach number of 0.65, which is the lower bound set in the optimization. The 
reduced Mach number contributes to a significant fuel saving: $8.83 \mathrm{lb} / \mathrm{nmi}$ for the $\mathrm{LRC}$ mission versus $14.4 \mathrm{lb} / \mathrm{nmi}$ for the TRC mission. Since the aircraft's MTOW is determined based on the fuel required for the LRC mission, and reducing the MTOW also reduces the DOC of the TRC mission, it is beneficial to reduce the fuel required for the LRC mission regardless of the flight time needed. For the LRC mission, the cruise segments dominate the mission range, time and fuel burn. For the TRC mission, though the cruise segment still dominates the mission range, time, and fuel burn, the contribution from other segments, especially climb, is also significant. This observation further emphasizes the importance of considering the entire mission profile, instead of only cruise, to have an accurate estimate of the fuel burn of shorter range missions.

Table 8: Optimized TRC mission (500 nmi) details.

\begin{tabular}{clccccc}
\hline & Segment & $\begin{array}{c}\text { Altitude } \\
\mathrm{ft}\end{array}$ & $\begin{array}{c}\text { Speed } \\
\text { Mach/kt }\end{array}$ & $\begin{array}{c}\text { Range } \\
\mathrm{nm}\end{array}$ & $\begin{array}{c}\text { Time } \\
\text { min }\end{array}$ & $\begin{array}{c}\text { Fuel Burn } \\
\mathrm{bb}\end{array}$ \\
\hline 0 & Start/WarmUp & 0 & - & 0.0 & - & $805.64(11.21 \%)$ \\
1 & Taxi & 0 & - & 0.0 & - & $797.59(11.09 \%)$ \\
2 & Takeoff & 0 & - & 0.0 & - & $394.81(5.49 \%)$ \\
3 & Climb & $1500 \rightarrow 10000$ & 150 KIAS $\rightarrow 250$ KIAS & 13.4 & 3.7 & $408.79(5.69 \%)$ \\
4 & Climb & $10000 \rightarrow 33082$ & $290 \mathrm{KIAS}$ & 138.9 & 19.7 & $1670.22(23.23 \%)$ \\
5 & Climb & $33082 \rightarrow 34185$ & M0.81 & 25.1 & 3.2 & $222.46(3.09 \%)$ \\
6 & Cruise & 34185 & M0.81 & 234.4 & 30.1 & $1857.46(25.83 \%)$ \\
7 & Descent & $34185 \rightarrow 33082$ & M0.81 & 2.2 & 0.3 & $3.17(0.04 \%)$ \\
8 & Descent & $33082 \rightarrow 10000$ & 290 KIAS & 66.1 & 10.0 & $133.81(1.86 \%)$ \\
9 & Descent & $10000 \rightarrow 1500$ & 250 KIAS $\rightarrow 150$ KIAS & 19.9 & 5.2 & $81.75(1.14 \%)$ \\
10 & Landing & 0 & - & 0.0 & - & $222.57(3.10 \%)$ \\
11 & Taxi & 0 & - & 0.0 & - & $591.73(8.23 \%)$ \\
\hline TRC Mission Total & & & 500.0 & 72.3 & 7190.0 \\
\hline
\end{tabular}

Table 9: Optimized LRC mission (2 000 nmi) details.

\begin{tabular}{clccccc}
\hline & Segment & $\begin{array}{c}\text { Altitude } \\
\mathrm{ft}\end{array}$ & $\begin{array}{c}\text { Speed } \\
\text { Mach/kt }\end{array}$ & $\begin{array}{c}\text { Range } \\
\mathrm{nm}\end{array}$ & $\begin{array}{c}\text { Time } \\
\text { min }\end{array}$ & $\begin{array}{c}\text { Fuel Burn } \\
\mathrm{lb}\end{array}$ \\
\hline 0 & Start/WarmUp & 0 & - & 0.0 & - & $910.42(5.15 \%)$ \\
1 & Taxi & 0 & - & 0.0 & - & $901.31(5.10 \%)$ \\
2 & Takeoff & 0 & - & 0.0 & - & $446.15(2.53 \%)$ \\
3 & Climb & $1500 \rightarrow 10000$ & 150 KIAS $\rightarrow 250$ KIAS & 16.2 & 4.6 & $497.21(2.81 \%)$ \\
4 & Climb & $10000 \rightarrow 21810$ & 290 KIAS & 40.3 & 6.6 & $665.50(3.77 \%)$ \\
5 & Climb & $21810 \rightarrow 35000$ & M0.65 & 82.5 & 12.9 & $932.82(5.28 \%)$ \\
6 & Cruise & 35000 & M0.65 & 873.4 & 139.8 & $6313.45(35.74 \%)$ \\
7 & Climb & $35000 \rightarrow 37000$ & M0.65 & 20.3 & 3.3 & $184.95(1.05 \%)$ \\
8 & Cruise & 37000 & M0.65 & 873.4 & 140.6 & $5769.91(32.66 \%)$ \\
9 & Descent & $37000 \rightarrow 21810$ & M0.65 & 39.7 & 6.2 & $66.86(0.38 \%)$ \\
10 & Descent & $21810 \rightarrow 10000$ & 290 KIAS & 34.2 & 5.7 & $82.51(0.47 \%)$ \\
11 & Descent & $10000 \rightarrow 1500$ & 250 KIAS $\rightarrow 150$ KIAS & 19.9 & 5.2 & $81.75(0.46 \%)$ \\
12 & Landing & 0 & - & 0.0 & - & $222.57(1.26 \%)$ \\
13 & Taxi & 0 & - & 0.0 & - & $591.73(3.35 \%)$ \\
\hline LRC Mission Total & & & 2000.0 & 324.8 & 17667.1 \\
\hline
\end{tabular}

The optimized DOC (based on the TRC mission) is tabulated in Table 10, broken down to its component costs. The component cost is sorted in a descending order. The highest contributor for DOC is fuel cost. By looking at Table 8, relatively high percentages of fuel burn are observed in the startup, taxi, takeoff, and landing segments. In fact, the total fuel burn from those segments amount to $39 \%$ of the total fuel burn for the $500 \mathrm{nmi}$ mission. The fuel burn of those segments are estimated using the fuel fraction method, with fuel fraction values taken from [13, 23, 24]. It is possible that the fuel fraction values are on the high side, which results in more fuel burn than they actually should be. Note that the mission range and time during those segments are not counted in getting the mission total values. These "extra" fuel burn, without any additional mission time, might contribute to the fuel burn dominating the DOC computation, as we observe.

Figure 9 shows the convergence history of the merit function, feasibility, and optimality, as defined in SNOPT [47]. 


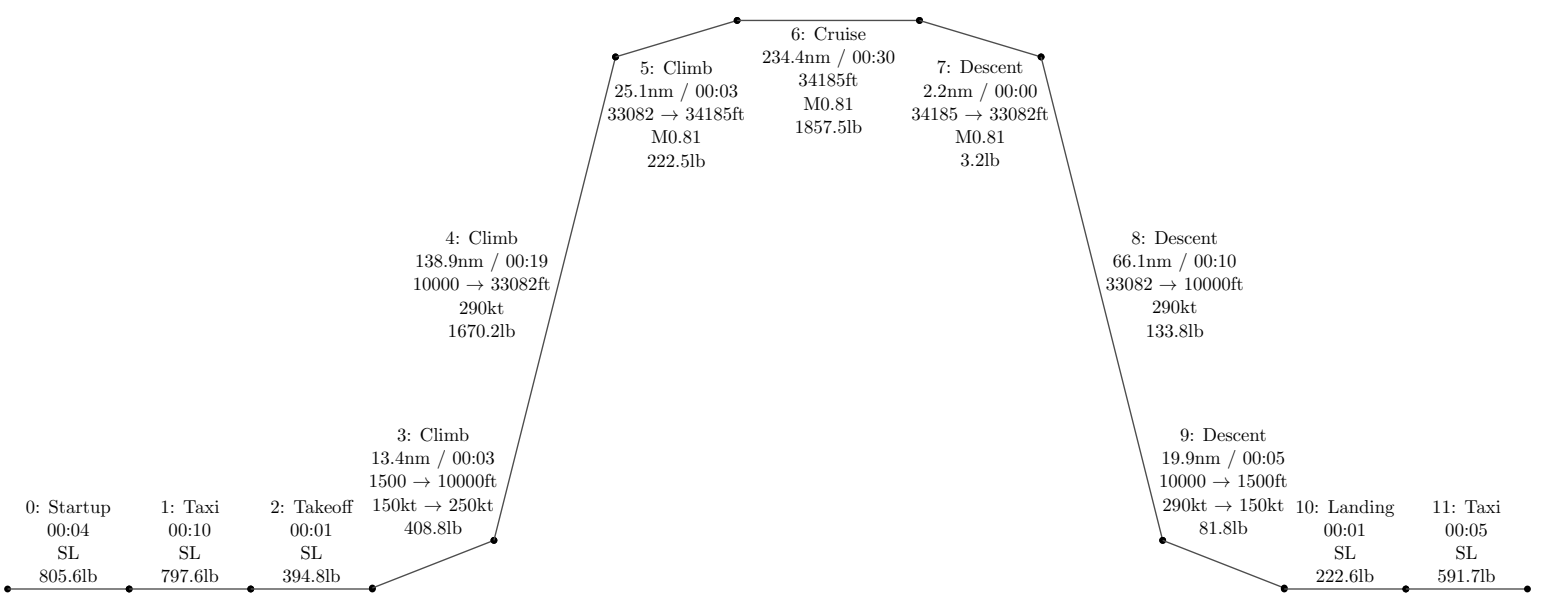

(a) TRC mission (500 nmi)

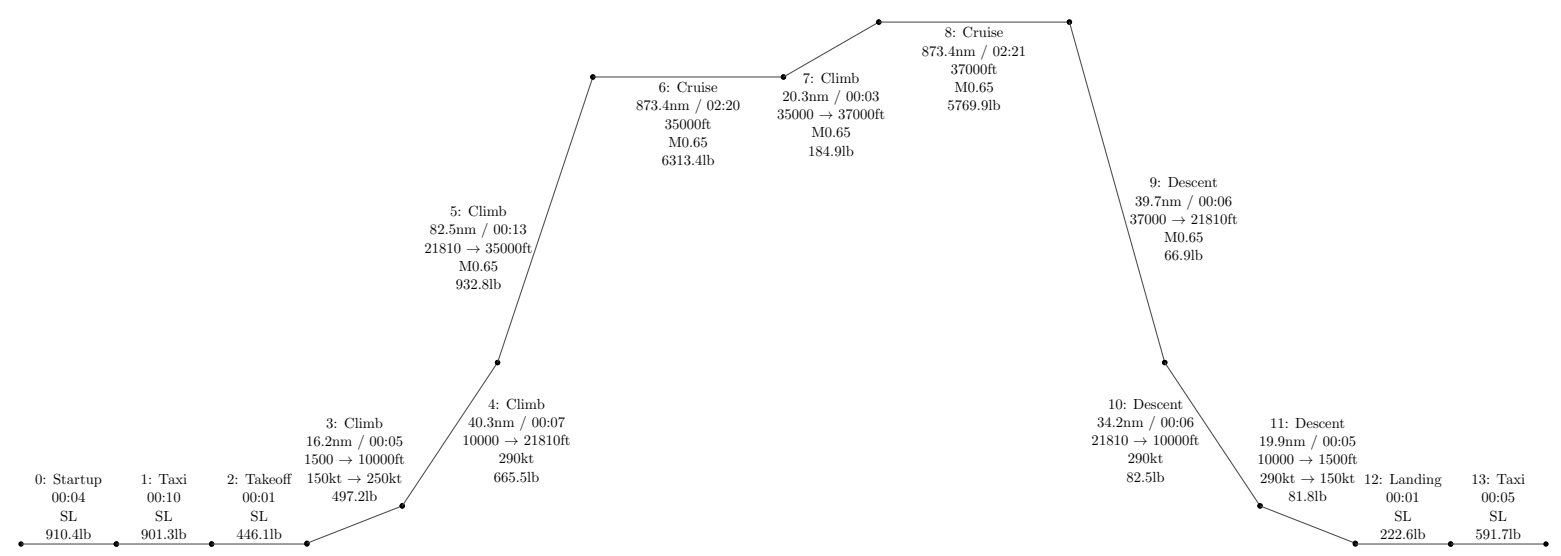

(b) LRC mission (2 $000 \mathrm{nmi})$

Figure 8: Optimal mission profiles for the TRC and LRC missions.

The optimality for this optimization problem is set at $2 \times 10^{-5}$. For this optimization problem, the optimality is achieved in approximately 90 mins, using only one processor.

\section{B. Aerostructural optimization results}

The results of the aerostructural optimization (35) are presented and discussed here. The aerostructural optimization is performed to minimize DOC, using five twist variables along the span as geometric design variables, with the wing planform geometry fixed. Wing twist is allowed to vary up to $\pm 10^{\circ}$, with the initial wing being untwisted. The optimized mission profile from the previous section is used, and this mission is assumed fixed throughout the optimization. This optimization results in a solution with all constraints satisfied. Between the mission profile and aerostructural optimizations, the aircraft MTOW increases by $3400 \mathrm{lb}(3.8 \%)$. This increase comes mainly from the additional constraints imposed on the aerostructural optimization. MTOW has a positive correlation with DOC, that is, an increase in MTOW will increase DOC, with other variables held constant. In our results, the overall DOC still decreases by $1 \%$ despite the increase in MTOW. The optimum DOC from the mission profile optimization results is $\$ 11390.86$ USD, whereas the aerostructural optimized DOC is \$11 274.37 USD. The breakdown and comparison of the DOC are shown in Table 10, and the improvement in DOC is due mostly from the fuel burn reduction of $207 \mathrm{lb}$.

The optimized thickness distribution is shown in Figure 10, with the insets showing the planform view of the wing structure as well as the wing deflection at $2.5 \mathrm{~g}$ MTOW. Most of the tail structure is at the minimum thickness bound, as well as the outer part of the wing and the ribs. The stringers that do not have their thicknesses linked to the skin panels are also at the minimum thickness bound, illustrating that the stringers would not have been correctly sized without considering buckling. The thickest part of the structure is at the root of the rear spar, as well as the location where the auxiliary spar attaches to the rear spar. The stress distribution of the $2.5 \mathrm{~g}$ MTOW load case, normalized by the lesser of the ultimate stress with a safety factor of 1.5 or the yield stress, is shown in Figure 11, with 
Table 10: Direct operating cost (\$USD) breakdown from mission optimization and aerostructural optimization.

\begin{tabular}{lcccc}
\hline Item & \multicolumn{2}{c}{ Mission Opt } & \multicolumn{2}{c}{ Aerostructural Opt } \\
\hline Fuel & $\$ 3308.48$ & $(29.05 \%)$ & $\$ 3101.63$ & $(27.51 \%)$ \\
Airport Fees & $\$ 2670.30$ & $(23.44 \%)$ & $\$ 2678.96$ & $(23.76 \%)$ \\
Airframe Maintenance & $\$ 1671.08$ & $(14.67 \%)$ & $\$ 1692.19$ & $(15.01 \%)$ \\
Financing & $\$ 733.94$ & $(6.44 \%)$ & $\$ 756.99$ & $(6.71 \%)$ \\
Depreciation & $\$ 685.62$ & $(6.02 \%)$ & $\$ 702.38$ & $(6.23 \%)$ \\
Navigation Fees & $\$ 633.70$ & $(5.56 \%)$ & $\$ 643.62$ & $(5.71 \%)$ \\
Crew & $\$ 543.82$ & $(4.77 \%)$ & $\$ 547.17$ & $(4.85 \%)$ \\
Engine Maintenance & $\$ 491.31$ & $(4.31 \%)$ & $\$ 492.71$ & $(4.37 \%)$ \\
Oil and Lubricants & $\$ 234.20$ & $(2.06 \%)$ & $\$ 234.20$ & $(2.08 \%)$ \\
Attendants & $\$ 231.39$ & $(2.03 \%)$ & $\$ 232.04$ & $(2.06 \%)$ \\
Insurance & $\$ 165.27$ & $(1.45 \%)$ & $\$ 170.54$ & $(1.51 \%)$ \\
Registry Taxes & $\$ 21.76$ & $(0.19 \%)$ & $\$ 21.92$ & $(0.19 \%)$ \\
\hline Total DOC & $\$ 11390.86$ & $(100.00 \%)$ & $\$ 11274.37$ & $(100.00 \%)$ \\
\hline
\end{tabular}

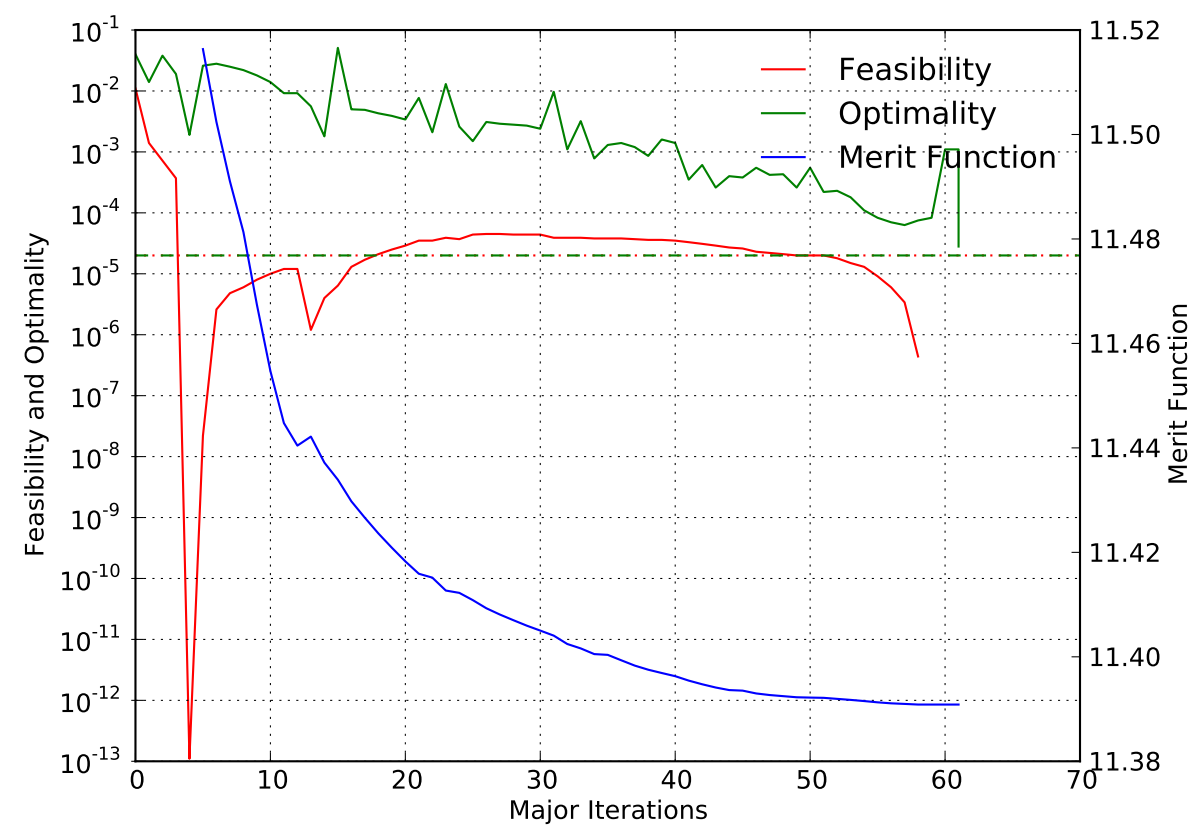

Figure 9: Convergence history of the merit function, feasibility, and optimality for the mission profile optimization. The optimality is set at $2 \times 10^{-5}$.

the insert showing the $C_{p}$ distribution over the top surface. This stress distribution shows that all stress constraints are satisfied, with the normalized stress being less than 1.0. Here we do not see many elements with active stress constraints (indicated by red color in the contour plot). Since we are using second-order elements in the finite element solver, stress singularities exist within poorly conditioned elements, causing unusually high stresses. These areas of high stress drive the optimization constraints to be active, while keeping other stresses way below the limit. These stress singularities can be eliminated with the use of higher-order elements. The optimized wing has a total weight of $9586.5 \mathrm{lb}$, which includes an estimate of the weight of the high-lift devices, as well as an empirical correction factor to account for details such as rivets, which are not modelled; while the total tail weight is estimated at $952.0 \mathrm{lb}$. A detailed weight comparison is shown in Table 11. The structural weights, combined with a required $17667 \mathrm{lb}$ of fuel required for the $2000 \mathrm{nmi}$ range, gives a total aircraft MTOW of $94450 \mathrm{lb}$, a $7 \%$ reduction compared to the initial MTOW of $101325 \mathrm{lb}$.

The spanwise lift, twist, and thickness-to-chord $(t / c)$ distributions for the $2.5 \mathrm{~g} \mathrm{MTOW}, \mathrm{ZFW}$, as well as the cruise 


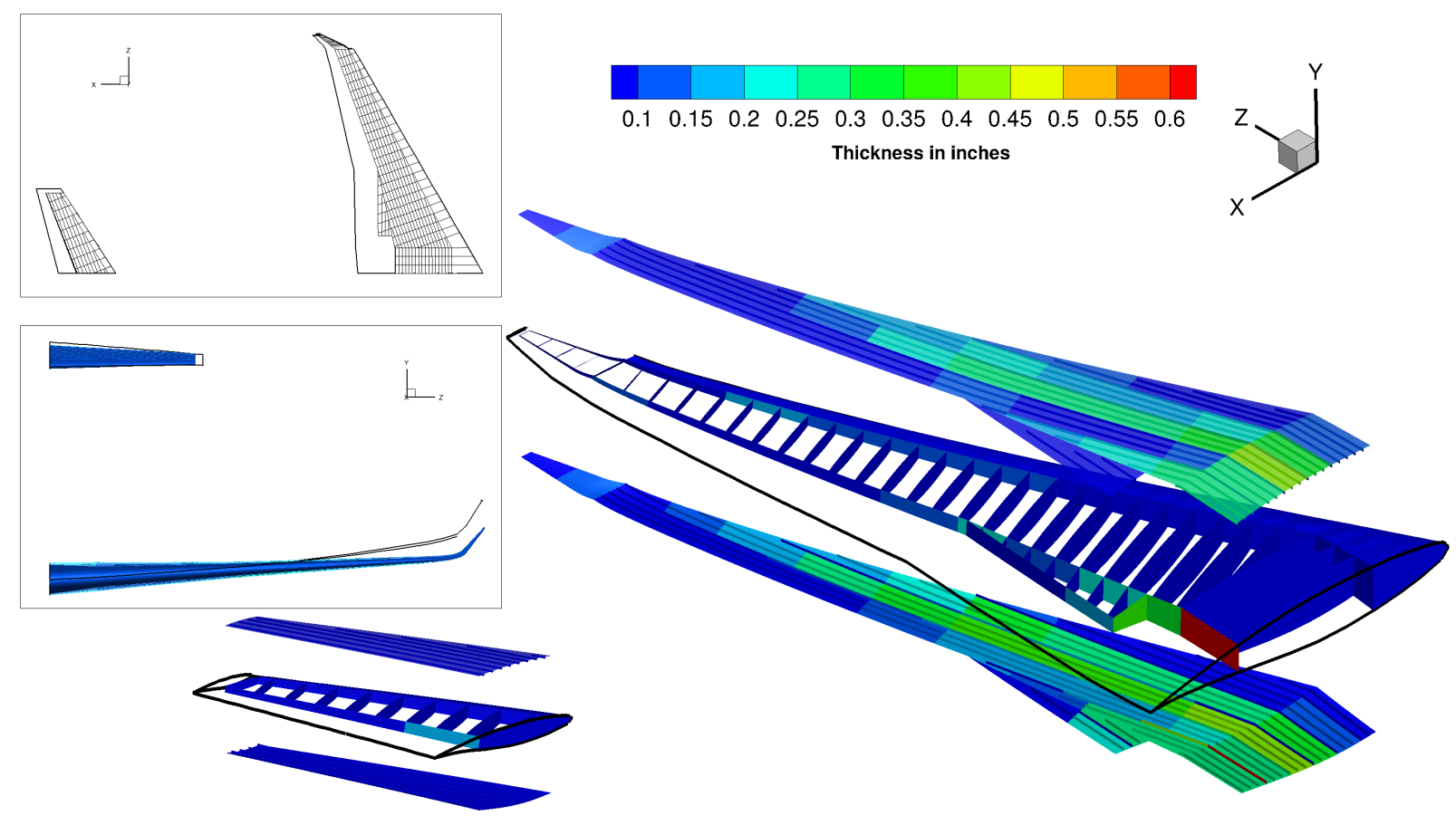

Figure 10: Thickness distribution of the aerostructural twist optimized structure, with the wing structural layout shown in the inset.

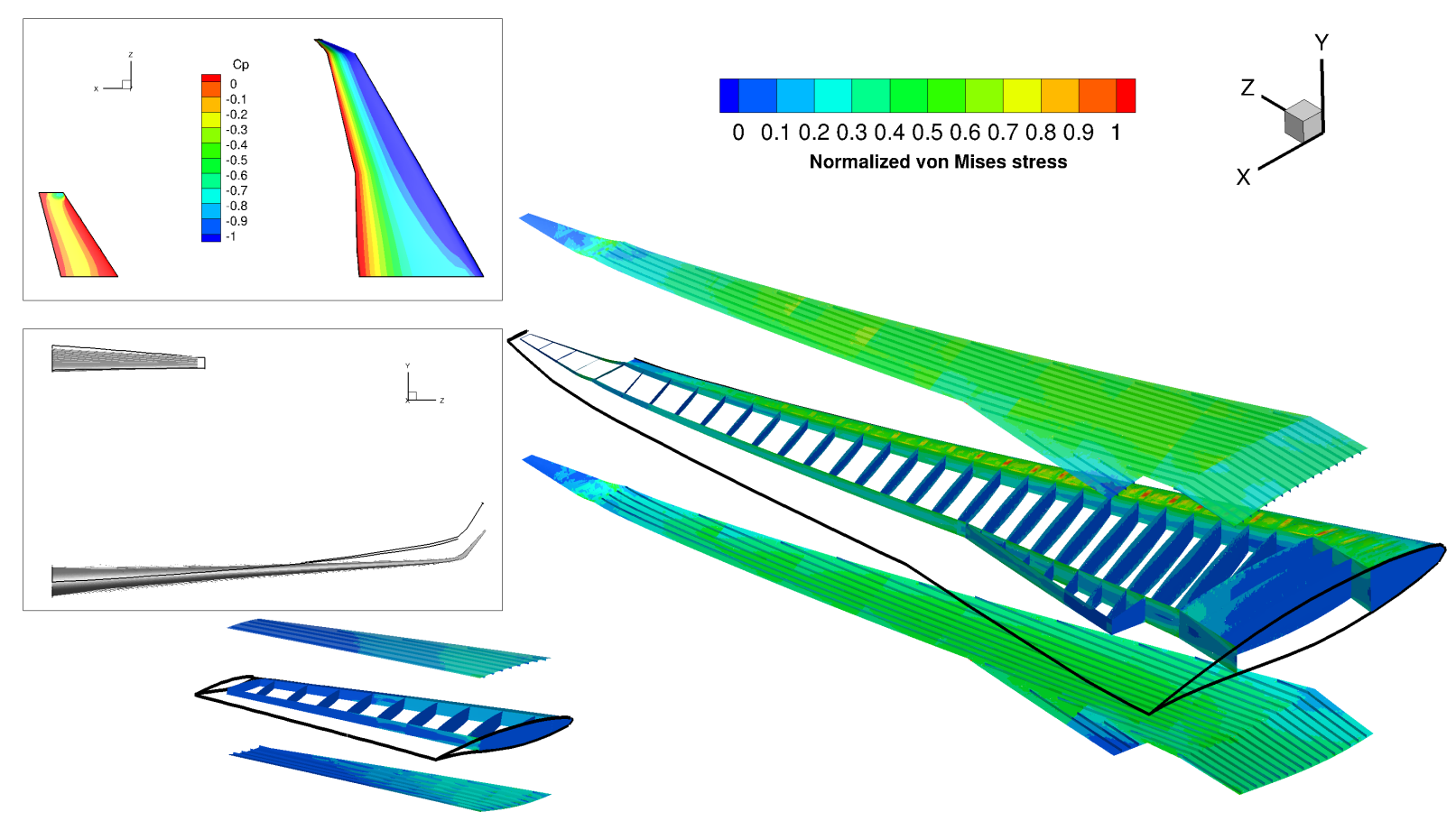

Figure 11: Stress distribution of the aerostructural twist optimized structure, showing the $2.5 \mathrm{~g}$ MTOW load cases, with the deformed flying wing shape shown in gray. 
Table 11: Detail of the wing and tail weight (lb) calculation, including a breakdown of the empirically corrected FEM weight and leading and trailing edge high-lift devices weight.

\begin{tabular}{rrr}
\hline Component & Wing & Tail \\
\hline Ribs & 413.5 & 47.8 \\
Spars & 325.8 & 28.0 \\
Top Skin/Stringers & 839.3 & 80.6 \\
Bottom Skin/Stringers & 1071.3 & 78.6 \\
\hline Wing Box (corr.) & 2647.7 & 234.9 \\
LE Device Weights & 452.4 & 42.7 \\
TE Device Weights & 1163.6 & 151.4 \\
\hline Total Wing Weight $(\times 2)$ & 9586.5 & 952.0 \\
\hline
\end{tabular}

load cases are shown in Figure 12. Compared to a reference elliptical distribution, the aerodynamic load for all the load cases are shifted slightly inboard, which is expected for an aerostructural optimization. The inboard shifting of the lift distribution reduces the bending moment at the wing root, thus resulting in a lighter structure than would otherwise be possible. The twist distributions also show that as the load is increased, the outer portion of the wing is twisted downwards (downwash), with the most significant twist from the $2.5 \mathrm{~g}$ MTOW case. Note that the upward inflections at the tip observed in the twist distribution correpond to the winglets. The $t / c$ distribution shows a linear decrease in thickness from root to the winglet, starting at $14 \%$ at the root, decreasing to an $11 \% t / c$ for the winglet.

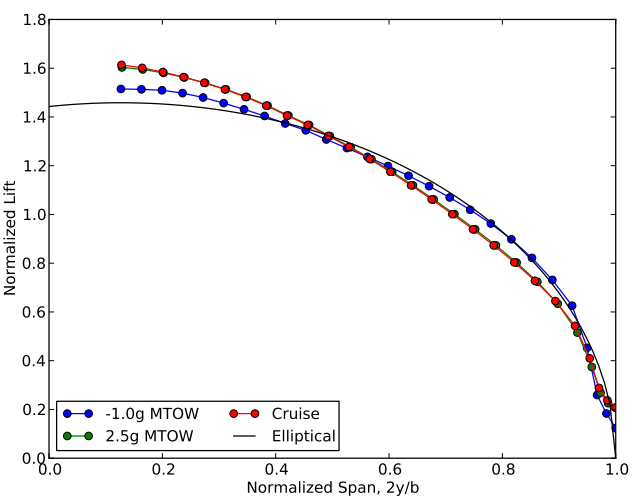

(a) Lift distribution

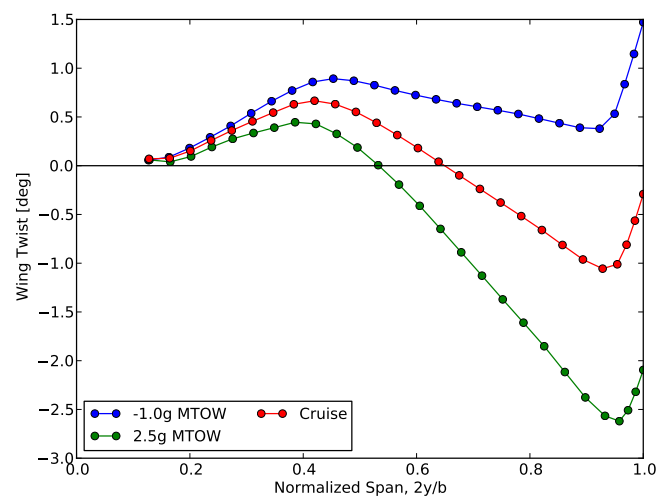

(b) Twist distribution

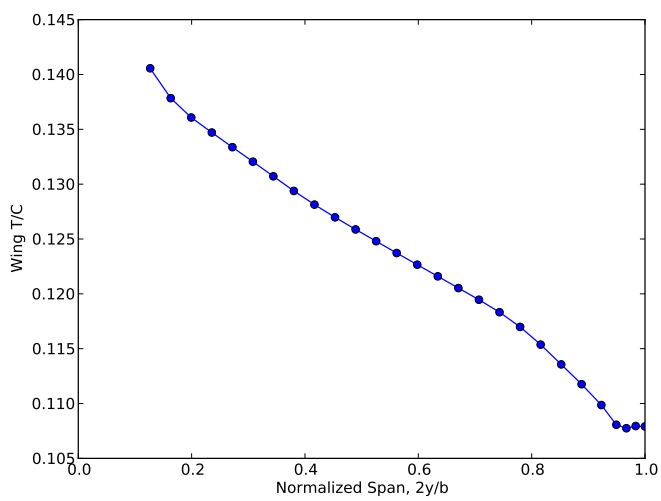

(c) Thickness distribution

Figure 12: Normalized lift, twist, and $t / c$ distribution of the aerostructural optimization.

Figure 13 shows the convergence history of the merit function, feasibility, and optimality for the aerostructural 
optimization case. The optimization is run with 96 processors for 90 hours and completes in 360 iterations. The optimality is reduced by six orders of magnitude from the first feasible point (at iteration 13) to the final (optimized) point. The merit function decreases monotonically, with a more rapid decrease between the $50^{\text {th }}$ and $120^{\text {th }}$ iteration.

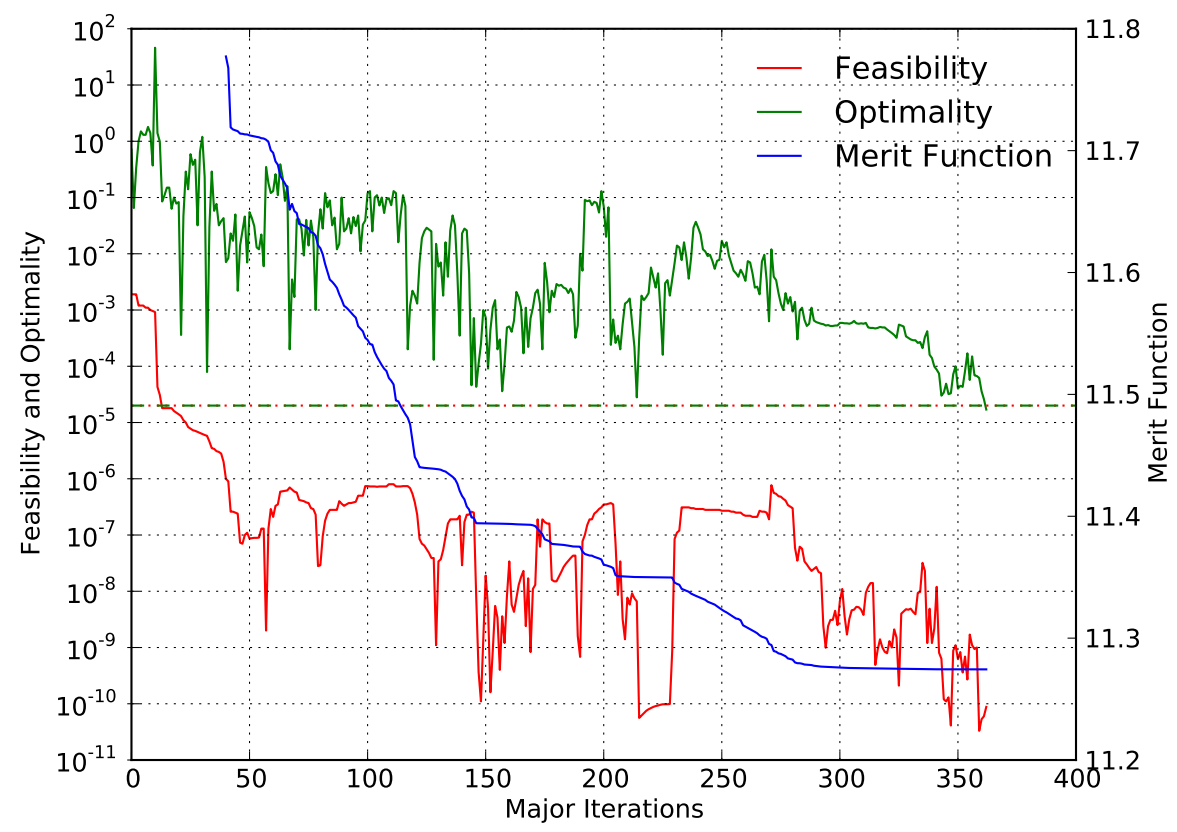

Figure 13: Convergence history of the merit function, feasibility, and optimality for the aerostructural optimization.

\section{Conclusion and future work}

In this paper, we present a DOC minimization case of a 100-passenger regional jet aircraft at its typical mission range of $500 \mathrm{nmi}$, while ensuring that the aircraft can perform and carry the full fuel required for the maximum range mission of $2000 \mathrm{nmi}$. The mission profile parameters (cruise Mach number and altitude) for each mission are determined by performing a mission profile optimization to minimize DOC, assuming a fixed aircraft geometry. These optimum mission profiles are used in the aerostructural optimization to optimize the aircraft geometry. To compute DOC, it is critical to have accurate computation of fuel burn, mission range and time. For this purpose, we develop an all-at-once mission analysis approach, which can model all flight segments (climb, cruise, and descent), and solve each segment independently. Detailed numerical integrations are performed within each segment to evaluate the total segment fuel burn, range, and time. Engine models are used to obtain TSFC values that accurately reflect the actual engine performance at different flight operating points in the mission, instead of assuming a constant value. The weights and CG locations of aircraft components are computed using a weight and balance model, and they are updated for each flight operating point to account for the decrease in weight due to fuel burn. With this approach, we have a more realistic analysis of the mission performance, without simplifying the physics of aircraft operations (e.g., assuming constant TSFC and speed), which is typical in empirical or analytical models. To make this complex analysis computationally tractable, we use kriging surrogate models to approximate the aerodynamic force and moment coefficients $\left(C_{L}, C_{D}\right.$, and $C_{m}$ ) in the space of Mach number, altitude, angle of attack, and tail rotation angle. By doing so, we reduce the number of aerodynamic solves required to complete the mission analysis from thousands to just 28 samples required to build kriging models. Constructing the kriging models with limited samples is challenging in this case because the kriging models need to cover the design space of the entire mission. This issue is more prominent in modeling $C_{D}$, due to the high drag gradient in the high Mach, high angle of attack region. We show in this work that by carefully specifying the basis functions for the kriging's global model (the deterministic term, or the function mean), the kriging model can now follow the underlying trend of $C_{D}$, and gives a better approximation without additional samples.

The results show that with the optimum twist distribution, the aerostructural optimization further reduces fuel burn by $207 \mathrm{lb}$ from the mission profile optimization results. This fuel burn saving leads to a net decrease in DOC of $1 \%$, despite an increase in aircraft MTOW of 3.8\%. In the solution, the range, stress, lift, and stability constraints are all satisfied for all flight conditions considered. This aerostructural optimization is run by using 96 processors and 
takes 90 hours to achieve the specified optimality and feasibility tolerances. Without using kriging surrogate models in the mission analysis procedure, the required computational time and resources will be significantly larger, due to the thousands of aerodynamic solves required at each iteration.

Having demonstrated the capability to optimize the mission profile as well as performing mission analyses for the aerostructural optimization, the next step is to use the module to simultaneously optimize the aircraft geometry and the mission profile in a coupled fashion. The use of surrogate models in the mission analysis offers the flexibility of using higher fidelity aerodynamics solver, such as Euler or RANS solvers, for more accurate results.

\section{Acknowledgments}

The authors are grateful for the funding provided by the Vanier Canada Graduate Scholarship (Natural Sciences and Engineering Research Council division), Zonta International Amelia Earhart Fellowships, and the Green Aviation Research and Deveplopment Network (GARDN) in collaboration with Bombardier Aerospace. Computations were performed on the GPC supercomputer at the SciNet HPC Consortium. SciNet is funded by: the Canada Foundation for Innovation under the auspices of Compute Canada; the Government of Ontario; Ontario Research Fund - Research Excellence; and the University of Toronto.

\section{References}

[1] ICAO. Aviation and Climate Change. International Civil Aviation Organization (ICAO) Environmental Report, 2010. URL http: //www.icao.int/environmental-protection/Pages/EnvReport10.aspx. (Accessed August 2013).

[2] International Energy Agency (IEA). World Energy Outlook 2008. International Energy Agency, Paris, France, 2008.

[3] J.J. Lee. Historical and Future Trends in Aircraft Performance, Cost, and Emissions. Master's thesis, Aeronautics \& Astronautics Department and Technology \& Policy Program, Massachusetts Institute of Technology, September 2000.

[4] N.E. Antoine and I.M. Kroo. Aircraft Optimization for Minimal Environmental Impact. Journal of Aircraft, 41(4):790-797, July-August 2004. doi:10.2514/1.71.

[5] K. Chiba, S. Obayashi, K. Nakahashi, and H. Morino. High-Fidelity Multidisciplinary Design Optimization of Wing Shape for Regional Jet Aircraft. In C.A. Coello Coello, A.H. Aguirre, and E. Zitzler, editors, Evolutionary Multi-Criterion Optimization. Third International Conference, EMO 2005, volume 3410, pages 621-635. Springer. Lecture Notes in Computer Science, Guanajuato, México, March 2005.

[6] A. Diedrich, J. Hileman, D. Tan, K. Willcox, and Z. Spakovsky. Multidisciplinary Design and Optimization of the Silent Aircraft. In 44th AIAA Aerospace Sciences Meeting and Exhibit, Reno, NV, January 9-12 2006. doi:10.2514/6.2006-1323. AIAA 2006-1323.

[7] S. Wakayama. Blended-Wing-Body Optimization Problem Setup. 2000. doi:10.2514/6.2000-4740. AIAA $2000-4740$.

[8] R.H. Liebeck. Design of the Blended Wing Body Subsonic Transport. Journal of Aircraft, 41(1):10-25, January 2004. doi:10.2514/1.9084.

[9] Gaetan K. W. Kenway, Graeme J. Kennedy, and Joaquim R. R. A. Martins. A scalable parallel approach for high-fidelity steady-state aeroelastic analysis and derivative computations. AIAA Journal, 2013. (In press).

[10] G.J. Kennedy and J.R.R.A. Martins. A Comparison of Metallic and Composite Aircraft Wings Using Aerostructural Design Optimization. In 14th AIAA/ISSMO Multidisciplinary Analysis and Optimization Conference, Indianapolis, IN, sep 2012. doi:10.2514/6.2012-5475. AIAA 2012-5475.

[11] Joaquim R. R. A. Martins and Andrew B. Lambe. Multidisciplinary design optimization: A survey of architectures. AIAA Journal, 2013. doi:10.2514/1.J051895. (In press).

[12] W.E. Randle, C.A. Hall, and M. Vera-Morales. Improved Range Equation Based on Aircraft Flight Data. Journal of Aircraft, 48(4):1291-1298, July-August 2011. doi:10.2514/1.C031262.

[13] J. Roskam. Airplane Design Part I: Preliminary Sizing of Airplanes. Roskam Aviation and Engineering Corporations, Ottawa, KS, 1985.

[14] J.G. Coffin. A Study of Airplane Range and Useful Loads. NACA-TR-69, NACA, 1920.

[15] L. Breguet. Calcul du Poids de Combustible Consummé par un Avion en Vol Ascendant. Comptes Rendus Hebdomodaires des Séances de l'Académie des Sciences, 177:870-872, 1923.

[16] B.W. McCormick. Aerodynamics, Aeronautics, and Flight Mechanics. John Wiley \& Sons, New York, US, 1979.

[17] I.M. Kroo. Aircraft Design: Synthesis and Analysis. Desktop Aeronautics, Palo Alto, CA, 1st edition, Sept 2006.

[18] H. Lee and G.B. Chatterji. Closed-Form Takeoff Weight Estimation Model for Air Transportation Simulation. In 10th AIAA Aviation Technology, Integration, and Operations (ATIO) Conference, Fort Worth, TX, Sept 13-15 2010. doi:10.2514/6.20109156. AIAA 2010-9156.

[19] Ryan P. Henderson, Joaquim R. R. A. Martins, and Ruben E. Perez. Aircraft conceptual design for optimal environmental performance. The Aeronautical Journal, 116:1-22, 2012.

[20] PASS. Program for aircraft synthesis studies software package. Desktop Aeronautics, Inc., Palo Alto, CA, 2005. URL http: //www. desktop.aero/pass.php.

[21] Benjamin Yan, Peter W. Jansen, and Ruben E. Perez. Multidisciplinary Design Optimization of Airframe and Trajectory Considering Cost and Emissions. In $14^{\text {th }}$ AIAA/ISSMO Multidisciplinary Analysis and Optimization (MAO) Conference, Indianapolis, IN, September 2012. doi:10.2514/6.2012-5494. AIAA 2012-5494. 
[22] R.P. Liem, G.K.W. Kenway, and J.R.R.A Martins. Multi-point, multi-mission, high-fidelity aerostructural optimization of a long-range aircraft configuration. In $14^{\text {th }}$ AIAA/ISSMO Multidisciplinary Analysis and Optimization (MAO) Conference, Indianapolis, IN, September 2012. doi:10.2514/6.2012-5706. AIAA 2012-5706.

[23] Daniel P. Raymer. Aircraft Design: A Conceptual Approach. Education Series. AIAA, Washington, DC, 1992.

[24] Mohammad H. Sadraey. Aircraft Design: A Systems Engineering Approach. John Wiley \& Sons, Chichester, West Sussex, 2012.

[25] J. Roskam and C.-T.E. Lan. Airplane Aerodynamics and Performance. DARcorporation, Lawrence, KS, 1997.

[26] J. Sacks, W.J. Welch, T.J. Mitchell, and H.P. Wynn. Design and Analysis of Computer Experiments. Statistical Science, 4: 409-423, 1989. doi:10.1214/ss/1177012413.

[27] J.M. ver Hoef and N. Cressie. Multivariable Spatial Prediction. Mathematical Geology, 25(2):219-240, 1993. doi:10.1007/BF00893273.

[28] Z.-H. Han, S. Görtz, and R. Zimmermann. On Improving Efficiency and Accuracy of Variable-Fidelity Surrogate Modeling in Aero-data for Loads Context. In Proceeding of CEAS 2009 European Air and Space Conference, Manchester, UK, October 26-29 2009.

[29] E.H. Isaaks and R.M. Srivastava. An Introduction to Applied Geostatistics. Oxford Univ. Press, Oxford, 1989.

[30] T.W. Simpson, T.M. Mauery, J.J. Korte, and F. Mistree. Kriging Metamodels for Global Approximation in Simulation-Based Multidisciplinary Design Optimization. AIAA Journal, 39(12):2233-2241, 2001.

[31] A. G. Journel and M. E. Rossi. When Do We Need a Trend Model in Kriging? Mathematical Geology, 21(7):715-739, 1989. doi:10.1007/BF00893318.

[32] R. Zimmermann. Asymptotic Behavior of the Likelihood Function of Covariance Matrices of Spatial Gaussian Processes. Journal of Applied Mathematics, 2010. doi:10.115/2010/494070.

[33] R. Hooke and T.A. Jeeves. "Direct Search" Solution of Numerical and Statistical Problems. Journal of the Association for Computing Machinery, 8:212-229, 1961. doi:10.1145/321062.321069.

[34] V. Torczon. On the Convergence of Pattern Search Algorithms. SIAM Journal of Optimization, 7(1):1-25, February 1997.

[35] S. N. Lophaven, H. B. Nielsen, and J. Søndergaard. Aspects of the MATLAB toolbox DACE. Technical Report IMM-REP2002-13, Informatics and Mathematical Modelling, Technical University of Denmark, 2002.

[36] Z.-H. Han, R. Zimmermann, and S. Görtz. A New Cokriging Method for Variable-Fidelity Surrogate Modeling of Aerodynamic Data. In 48th AIAA Aerospace Sciences Meeting Including the New Horizons Forum and Aerospace Exposition, Orlando, FL, January 4-7 2010. doi:10.2514/6.2010-1225. AIAA 2010-1225.

[37] S.E. Gano, J.E. Renaud, J.D. Martin, and T.W. Simpson. Update strategies for kriging models used in variable fidelity optimization. Multidisciplinary Optimization, 32:287-298, 2006. doi:10.1007/s00158-006-0025-y.

[38] Metallic materials and elements for aerospace vehicle structures. Technical Report MIL-HDBK-5J, Department of Defense, 31 January 2003.

[39] R. Hangartner. Correlation of fatigue data for aluminum aircraft wing and tail structures. Technical Report 14555, National Research Council of Canada, December 1974.

[40] G.J. Kennedy and J.R.R.A. Martins. Parallel Solution Methods for Aerostructural Analysis and Design Optimization. In 13th AIAA/ISSMO Multidisciplinary Analysis and Optimization Conference, Fort Worth, TX, September 2010. doi:10.2514/6.2010-9308. AIAA 2010-9308.

[41] S. A. Brown. Displacement extrapolation for CFD+CSM aeroelastic analysis. In Proceedings of the 35th AIAA Aerospace Sciences Meeting, Reno, NV, 1997. doi:10.2514/6.1997-1090. AIAA-97-1090.

[42] Joaquim R. R. A. Martins, Juan J. Alonso, and James J. Reuther. A Coupled-Adjoint Sensitivity Analysis Method for High-Fidelity Aero-Structural Design. Optimization and Engineering, 6(1):33-62, March 2005. doi:10.1023/B:OPTE.0000048536.47956.62.

[43] Gaetan K. W. Kenway and Joaquim R. R. A. Martins. Multi-point High-fidelity Aerostructural Optimization of a Transport Aircraft Configuration. Journal of Aircraft, 2013. doi:10.2514/1.C032150. (In press).

[44] J. Roskam. Airplane Design Part VIII: Airplane Cost Estimation: Design, Development, Manufacturing and Operating. Roskam Aviation and Engineering Corporations, Ottawa, KS, 1990.

[45] The GasTurb Program: http://www. gasturb. de (Accessed August 2013).

[46] L. Hascoët. Tapenade: A tool for automatic differentiation of programs. Proceedings of $4^{\text {th }}$ European Congress on Computational Methods, ECCOMAS'2004, Jyvaskyla, Finland, 2004.

[47] Philip E. Gill, Walter Murray, and Michael A. Saunders. SNOPT: An SQP Algorithm for Large Scale Constrained Optimization. SIAM Review, 47(1):99-131, 2005. doi:10.1137/S0036144504446096.

[48] Ruben Perez, Peter Jansen, and Joaquim R. R. A. Martins. pyOpt: a Python-based object-oriented framework for nonlinear constrained optimization. Structural and Multidisciplinary Optimization, 45:101-118, 2011. ISSN 1615-147X. doi:10.1007/s00158-011-0666-3.

[49] Chris Loken, Daniel Gruner, Leslie Groer, Richard Peltier, Neil Bunn, Michael Craig, Teresa Henriques, Jillian Dempsey, Ching-Hsing Yu, Joseph Chen, L Jonathan Dursi, Jason Chong, Scott Northrup, Jaime Pinto, Neil Knecht, and Ramses Van Zon. Scinet: Lessons learned from building a power-efficient top-20 system and data centre. Journal of Physics: Conference Series, 256(1):012026, 2010. doi:10.1088/1742-6596/256/1/012026.

[50] Federal aviation regulations (FAR). Part 91 General Operating and Flight Rules, Federal Aviation Administration (FAA), 2012.

[51] Andrew B. Lambe and Joaquim R. R. A. Martins. Extensions to the design structure matrix for the description of multidisciplinary design, analysis, and optimization processes. Structural and Multidisciplinary Optimization, 46:273-284, August 2012. doi:10.1007/s00158-012-0763-y. 
[52] E. Torenbeek. Development and application of a comprehensive, design-sensitive weight prediction method for wing structures of transport category aircraft. Technical Report LR-693, Delft University of Technology, Netherlands, 1992.

[53] Federal aviation regulations (FAR). Part 25 Airworthiness Standards: Transport Category Airplanes, Federal Aviation Administration (FAA), 2012.

[54] Charles A. Mader and Joaquim R. R. A. Martins. Optimal flying wings: A numerical optimization study. Journal of Aircraft, 2012. (Accepted subject to revisions).

[55] G.A. Wrenn. An indirect method for numerical optimization using the Kreisselmeier-Steinhauser function'. NASA Technical Report CR-4220, 1989.

[56] N.M.K. Poon and J.R.R.A. Martins. An adaptive approach to constraint aggregation using adjoint sensitivity analysis. Structures and Multidisciplinary Optimization, 30(1):61-73, 2007. doi:10.1007/s00158-006-0061-7. 